\title{
Global bifurcation of solutions of the mean curvature spacelike equation in certain Friedmann-Lemaitre-Robertson-Walker spacetimes
}

\author{
Guowei Dai $^{\star}$, Alfonso Romero ${ }^{\dagger}$ and Pedro J. Torres ${ }^{\ddagger} \dagger$ \\ * School of Mathematical Sciences, \\ Dalian University of Technology, Dalian, 116024, PR China \\ E-mail: daiguowei@dlut.edu.cn \\ † Departamento de Geometría y Topología, \\ Universidad de Granada, 18071 Granada, Spain \\ E-mail: aromero@ugr.es \\ ¥ Departamento de Matemática Aplicada, \\ Universidad de Granada, 18071 Granada, Spain \\ E-mail: ptorres@ugr.es
}

\begin{abstract}
We study the existence of spacelike graphs for the prescribed mean curvature equation in the Friedmann-Lemaitre-Robertson-Walker (FLRW) spacetime. By using a conformal change of variable, this problem is translated into an equivalent problem in the Lorentz-Minkowski spacetime. Then, by using Rabinowitz's global bifurcation method, we obtain the existence and multiplicity of positive solutions for this equation with 0-Dirichlet boundary condition on a ball. Moreover, the global structure of the positive solution set is studied.
\end{abstract}

Keywords: Bifurcation; Mean curvature spacelike equation; Positive solution; FriedmannLemaitre-Robertson-Walker spacetime

MSC(2000): 35B32; 53A10; 35B40; 53B30; 53C50

\footnotetext{
${ }^{*}$ Corresponding author.

${ }^{\dagger}$ The first author is supported by NNSF of China no. 11401477, the second one by Spanish MINECO Grant with FEDER funds MTM2016-78807-C2-1-P and the third author by Spanish MICINN Grant with FEDER funds MTM2014-52232-P.
} 


\section{Introduction and main results}

Let $I \subseteq \mathbb{R}$ be an open interval endowed with the (negative definite) metric $-d t^{2}$. Denote by $\mathcal{M}$ the $(N+1)$-dimensional product manifold $I \times \mathbb{R}^{N}$ with $N \geq 1$ endowed the Lorentzian metric

$$
g=-d t^{2}+f^{2}(t) d x^{2},
$$

where $f \in C^{\infty}(I), f>0$, is called the scale factor or warping function. Clearly, $\mathcal{M}$ is a Lorentzian warped product, in the sense of [21], with base $\left(I,-d t^{2}\right)$, fiber $\left(\mathbb{R}^{N}, d x^{2}\right)$ and warping function $f$. This type of spacetimes plays a central role in General Relativity. For $\operatorname{dim} \mathcal{M}=4, t$ may be interpreted as the relative time of a family of privileged observers, the so-called co-moving observers, and for them the quantity $f(t)$ is the radius of their spatial universe at time $t$. Then, the positive (resp. negative) sign of $f^{\prime}(t)$ indicates that these observers perceive expansion (resp. contraction) at a given time $t$. Moreover, for warping functions close to 1 , the corresponding spacetime $\mathcal{M}$ may be thought as a deformation of the Lorentz-Minkowski spacetime, so these spacetimes are good candidates to explore stability of physical properties expressed for a empty universe in terms of the Lorentz-Minkowski spacetime. In this paper, we will refer $\mathcal{M}$ as a (flat fiber) Friedmann-Lemaître-RobertsonWalker (FLRW) spacetime. More generally, if the fiber of $\mathcal{M}$ is changed to an $N$-dimensional Riemannian manifold of constant sectional curvature we arrive to the notion of (general) FLRW spacetime.

In the four dimensional case, FLRW spacetimes have been useful to obtain exact solutions of Einstein's field equations of General Relativity because they describe spatially homogeneous and isotropic (expanding or contracting) universes. These geometric properties are in complete agreement with the experience and, therefore, these models have been useful to describe the large scale of the universe from the point of view of the relativistic cosmology. The pioneering and important results obtained by the use of FLRW models were first derived by Friedmann in 1922 and 1924 [14, 15]. In 1927, Lemaître [18] arrived independently at similar results as those of Friedmann. Robertson and Walker explored later the problem further during the 1930s [25, 26, 27, 30]. In particular, Robertson rigorously proved that a spatially homogeneous and isotropic spacetime must be locally isometric to a FLRW spacetime in 1935. For more details of FLRW spacetimes, see [21, Chapter 12] or the monograph of Choquet-Bruhat [9] and the references therein.

Given $f \in C^{\infty}(I), f>0$, for each $u \in C^{\infty}(\Omega)$, where $\Omega$ is a domain of $\mathbb{R}^{N}$, such that $u(\Omega) \subset I$ we can consider its graph $M=\{(u(x), x): x \in \Omega\}$ in the FLRW spacetime $\mathcal{M}$. The graph inherits a metric from (1.1), given by

$$
-d u^{2}+f^{2}(u) d x^{2}
$$

on $\Omega$, which is positive definite if and only if $u$ satisfies

$$
|\nabla u|<f(u)
$$

everywhere on $\Omega$, where $\nabla u$ is the gradient of $u$ in $\mathbb{R}^{N}$ and $|\nabla u|$ its lenght. When the metric (1.2) is Riemannian, the graph $M$ is called spacelike. In this case, the pointing future unitary normal vector field on $M$ is given by

$$
\frac{1}{f(u) \sqrt{f(u)^{2}-|\nabla u|^{2}}}\left(f^{2}(u), \nabla u\right),
$$


and the corresponding mean curvature function $H$ satisfies

$$
\operatorname{div}\left(\frac{\nabla u}{f(u) \sqrt{f^{2}(u)-|\nabla u|^{2}}}\right)+\frac{f^{\prime}(u)}{\sqrt{f^{2}(u)-|\nabla u|^{2}}}\left(N+\frac{|\nabla u|^{2}}{f^{2}(u)}\right)=N H,
$$

where div denotes the divergence operator of $\mathbb{R}^{N}$ and $f^{\prime}(u):=f^{\prime} \circ u$. Equation (1.5) and (1.3) is called the mean curvature spacelike equation in the FLRW spacetime $\mathcal{M}$. Specially relevant is the case when $H$ is constant, then it is called the constant mean curvature spacelike equation (the maximal graph equation if $H=0$ ).

In the related literature, most of the efforts have been directed to the case $f \equiv 1$ (Minkowsky spacetime). When $H \equiv 0$ and $\Omega=\mathbb{R}^{N}$, Calabi [7] proved that equation (1.5) under (1.3) has only linear entire solutions for $N \leq 4$. Further, Cheng and Yau [8] extended the result for all $N$. This striking result contrasts with the answer to the classical Bernstein conjecture for the minimal graph equation which states that the only entire solutions of the minimal graph equation in $\mathbb{R}^{N+1}$ are linear only for $N \leq 7,[22]$. When $f \equiv 1, \Omega=\mathbb{R}^{N}$ and $H$ is a positive constant, some celebrated results for equation (1.5) were obtained by Treibergs [29]. If $f \equiv 1, \Omega$ is a bounded $C^{2, \alpha}$ domain with some $\alpha>0$ and $H=H(u, x) \in C^{0, \alpha}(\mathbb{R} \times \Omega)$ is bounded, Bartnik and Simon [2] proved that equation (1.5) with $u=\varphi$ on $\partial \Omega$ has a strictly spacelike solution $u \in C^{2, \alpha}(\bar{\Omega})$, where $\varphi$ is bounded and has an extension $\bar{\varphi} \in C^{2, \alpha}(\bar{\Omega})$ satisfying $|\nabla \bar{\varphi}| \leq 1-\theta$ in $\bar{\Omega}$ for some $\theta>0$. More recently, Bereanu, Jebelean and Torres established in $[4,5]$ some nonexistence, existence and multiplicity results for positive radial solutions of equation (1.5) with $f \equiv 1$, $\Omega=B_{R}=B_{R}(0):=\left\{x \in \mathbb{R}^{N}:|x|<R\right\}$ with $R>0, u=0$ on $\partial \Omega$. Recently, when $f \equiv 1$, the first author [10] studied the nonexistence, existence and multiplicity of positive radial solutions of equation (1.5) on the unit ball with $u=0$ on $\partial B_{R}$ and $N H=-\lambda f(s, x)$ via bifurcation method [23, Theorem 1.3], which were extended to the general domain in [12].

Rather less attention has been paid to the case when $f$ is not a constant. Only in the recent years, the authors of $[3,16,20]$ have studied the existence of radially symmetric spacelike graphs by combining Leray-Schauder degree or the classical Schauder fixed Theorem with approximation processes.

To study equation (1.5), we follow the method developed in [3, 16, 20], namely, we introduce a conformal change of variables that lead to an equivalent prescribed mean curvature problem in the Lorentz-Minkowski spacetime (see (1.10)). Define the "conformal" time

$$
\varphi(t)=\int_{0}^{t} \frac{d \xi}{f(\xi)}
$$

for all $t \in I$. Clearly, $\varphi$ is strictly increasing with $\varphi(0)=0$. Therefore, it defines a diffeomorphism $\varphi: I \rightarrow J$, where $J$ is an open interval of $\mathbb{R}$ with $0 \in J$. Now, we can define a map $F: I \times \mathbb{R}^{N} \longrightarrow J \times \mathbb{R}^{N}$ by $F(t, x)=(\varphi(t), x)$, which is clearly a diffeomorphism and satisfies $F^{*}(\bar{g})=g$, where $g$ is the Lorentzian metric given by (1.1) and

$$
\bar{g}=f^{2}\left(\varphi^{-1}(s)\right)\left(-d s^{2}+d x^{2}\right),
$$

where $\varphi^{-1}$ is the inverse function of $\varphi$. By a simple computation we have

$$
\varphi^{-1}(s)=\int_{0}^{s} f\left(\varphi^{-1}(\xi)\right) d \xi
$$


In other words, $F$ is an isometry from $\left(I \times \mathbb{R}^{N}, g\right)$ onto $\left(J \times \mathbb{R}^{N}, \bar{g}\right)$. If as previously $M$ denotes the graph defined by $u$ then $F(M)$ is the graph in $J \times \mathbb{R}^{N}$ of the function $v$ given by

$$
v=\varphi(u) .
$$

From the previous formula we get $|\nabla u|=f(u)|\nabla v|$, and therefore, it follows that $M$ is spacelike if and only if $F(M)$ is spacelike, and in this case we have $|\nabla v|<1$. Moreover, it is not difficult to show that

$$
\operatorname{div}\left(\frac{\nabla u}{f(u) \sqrt{f^{2}(u)-|\nabla u|^{2}}}\right)=\frac{1}{f(u)} \operatorname{div}\left(\frac{\nabla v}{\sqrt{1-|\nabla v|^{2}}}\right)-\frac{f^{\prime}(u)|\nabla v|^{2}}{f(u) \sqrt{1-|\nabla v|^{2}}} .
$$

Therefore, $u \in C^{2}\left(B_{R}\right), u\left(B_{R}\right) \subset I$, where $B_{R}$ be the standard open ball in $\mathbb{R}^{N}$ which is centered at the origin and has radius $R$, under the constraint $|\nabla u|<f(u)$, is a solution of equation (1.5) if and only if $v, v\left(B_{R}\right) \subset J$, under the constraint $|\nabla v|<1$, is a solution of the equation

$$
\operatorname{div}\left(\frac{\nabla v}{\sqrt{1-|\nabla v|^{2}}}\right)+\frac{N f^{\prime}\left(\varphi^{-1}(v)\right)}{\sqrt{1-|\nabla v|^{2}}}=N f\left(\varphi^{-1}(v)\right) H\left(\varphi^{-1}(v), x\right) .
$$

The form of the previous equation suggests to consider the function $v$ as defining a spacelike graph in the product spacetime $\left(J \times \mathbb{R}^{N},-d s^{2}+d x^{2}\right)$, which is just an open subset of the $(N+1)$-dimensional Lorentz-Minkowski spacetime, with mean curvature

$$
-\frac{f^{\prime}\left(\varphi^{-1}(v)\right)}{\sqrt{1-|\nabla v|^{2}}}+f\left(\varphi^{-1}(v)\right) H\left(\varphi^{-1}(v), x\right) .
$$

We consider the following 0-Dirichlet boundary value problem

$$
\begin{cases}-\operatorname{div}\left(\frac{\nabla v}{\sqrt{1-|\nabla v|^{2}}}\right)=\lambda\left(\frac{N f^{\prime}\left(\varphi^{-1}(v)\right)}{\left.\sqrt{1-|\nabla v|^{2}}-N f\left(\varphi^{-1}(v)\right) H\left(\varphi^{-1}(v), x\right)\right)}\right. & \text { in } B_{R}, \\ v=0 & \text { on } \partial B_{R},\end{cases}
$$

where $\lambda$ is a real parameter, which can represent in some sense the strength of warping function, $H: I \times \bar{B}_{R} \rightarrow \mathbb{R}$ is a continuous function and is radially symmetric with respect to $x$.

The aim of this paper is to investigate the existence of positive radially symmetric spacelike solutions for problem (1.11) by means of the global bifurcation method.

Passing to polar coordinates, the problem (1.11) is reduced to the following ODE with mixed boundary conditions

$$
\left\{\begin{array}{l}
-\left(r^{N-1} \phi\left(v^{\prime}\right)\right)^{\prime}=\lambda N r^{N-1}\left(\frac{f^{\prime}\left(\varphi^{-1}(v)\right)}{\sqrt{1-v^{\prime 2}}}-f\left(\varphi^{-1}(v)\right) H\left(\varphi^{-1}(v), r\right)\right), r \in(0, R), \\
v^{\prime}(0)=v(R)=0,
\end{array}\right.
$$

where $r=|x|, \phi(s)=s / \sqrt{1-s^{2}}$. A solution $v$ of problem (1.12) is understood in the classical sense, i.e., it belongs to $C^{1}[0, R] \cap C^{2}(0, R)$ such that problem (1.12) is satisfied.

Since the graph associated to $v$ is spacelike, we deduce that $\|v\|_{\infty}<R$. So, the image of nonnegative $v$ lies in $[0, R]$. Hence, from now on, we always assume that $[0, R] \subset \varphi(I)$, which is equivalent to

$$
I_{f}(R):=\left[0, \int_{0}^{R} f\left(\varphi^{-1}(s)\right) d s\right] \subset I .
$$


It follows that $H$ is bounded.

Let $\lambda_{1}$ be the first eigenvalue of

$$
\left\{\begin{array}{l}
-\left(r^{N-1} u^{\prime}\right)^{\prime}=\lambda r^{N-1} u, \quad r \in(0, R), \\
u^{\prime}(0)=u(R)=0 .
\end{array}\right.
$$

Let

$$
X=\left\{v \in C^{1}[0, R]: v^{\prime}(0)=v(R)=0\right\}
$$

with the norm $\|v\|:=\left\|v^{\prime}\right\|_{\infty}$. From the fact $\|v\|_{\infty} \leq\left\|v^{\prime}\right\|_{\infty} R$, it is easy to verify that the norm $\|v\|$ is equivalent to the usual norm $\|v\|_{\infty}+\left\|v^{\prime}\right\|_{\infty}$. For any $A \subseteq \mathbb{R} \times X$, we use $\operatorname{pr}_{\mathbb{R}}(A)$ denoting the projection of $A$ on $\mathbb{R}$.

The following theorem is our main result.

Theorem 1.1. Assume that $f^{\prime}(t) \geq 0$ for any $t \in I_{f}(R)$ and $f^{\prime}(t)>f(t) H(t, r)$ for any $r \in[0, R], t \in I_{f}(R) \backslash\{0\}$, and there exist $\left.\left.f_{0} \in[0,+\infty], H_{0} \in\right]-\infty,+\infty\right]$ with $f_{0}+H_{0} \in[0,+\infty]$ such that

$$
\lim _{t \rightarrow 0^{+}} \frac{N f^{\prime}(t)}{\varphi(t)}=f_{0}, \quad \lim _{t \rightarrow 0^{+}} \frac{N f(t) H(t, r)}{\varphi(t)}=-H_{0}
$$

uniformly for $r \in(0, R)$. Then,

(a) if $f_{0}+H_{0}=1$, there is an unbounded component $\mathscr{C}$ of the set of positive solutions of problem (1.12) bifurcating from $\left(\lambda_{1}, 0\right)$ such that $\mathscr{C} \subseteq\left(\left(\left(\mathbb{R}_{+} \backslash\{0\}\right) \times X\right) \cup\left\{\left(\lambda_{1}, 0\right)\right\}\right)$, $\left(\lambda_{1},+\infty\right) \subseteq p r_{\mathbb{R}}(\mathscr{C}),\left\|v_{\lambda}\right\|<1$ and $\lim _{\lambda \rightarrow+\infty}\left\|v_{\lambda}\right\|=1$ for $\left(\lambda, v_{\lambda}\right) \in \mathscr{C} \backslash\left\{\left(\lambda_{1}, 0\right)\right\}$,

(b) if $f_{0}+H_{0}=+\infty$, there is an unbounded component $\mathscr{C}$ of the set of positive solutions of problem (1.12) emanating from $(0,0)$ and joining to $(+\infty, 1)$ such that $\mathscr{C} \subseteq$ $\left(\left(\left(\mathbb{R}_{+} \backslash\{0\}\right) \times X\right) \cup\{(0,0)\}\right)$ and $\left\|v_{\lambda}\right\|<1$ for any $\left(\lambda, v_{\lambda}\right) \in \mathscr{C} \backslash\{(0,0)\}$ with $\lambda<+\infty$,

(c) if $f_{0}+H_{0}=0$, there is an unbounded component $\mathscr{C}$ of the set of positive solutions of problem (1.12) in $\mathbb{R}_{+} \times X$ which joins $(+\infty, 1)$ to $(+\infty, 0)$ and $\left\|v_{\lambda}\right\|<1$ for any $\left(\lambda, v_{\lambda}\right) \in \mathscr{C}$ with $\lambda<+\infty$.

Following [24], we add the point $\infty$ to our space $\mathbb{R} \times X$ so that $(+\infty, 1)$ and $(+\infty, 0)$ are elements of $\mathscr{C}$. Figure 1 illustrates the global bifurcation branches of Theorem 1.1. It follows from Theorem 1.1 that problem (1.12) possesses at least one positive solution for any $\lambda \in\left(\lambda_{1},+\infty\right)$ if $f_{0}+H_{0}=1$, and has at least one positive solution for any $\lambda \in(0,+\infty)$ if $f_{0}+H_{0}=+\infty$, see (a) and (b) of Figure 1. Moreover, when $f_{0}+H_{0}=0$, there exists $\lambda_{*}>0$ such that problem (1.12) has at least two positive solutions for any $\lambda \in\left(\lambda_{*},+\infty\right)$, see (c) of Figure 1. As a further remark, let us note that a simple rescaling shows that if $0<f_{0}+H_{0}<+\infty$, then there is a branch emanating from with the $\left(\lambda_{1} /\left(f_{0}+H_{0}\right), 0\right)$ with the same properties as in Theorem 1.1 (a).

The remainder of this paper is arranged as follows. In Section 2, we study the global bifurcation phenomenon of an approximation problem. Section 3 is devoted to prove Theorem 1.1. Finally, for the sake of completeness an Appendix is included in Section 4, where a detailed derivation of the mean curvature spacelike equation (1.5) is given. 


\section{Global bifurcation for an approximation problem}

Expanding the left member of the first equation of problem (1.12), we have that

$$
-\frac{v^{\prime \prime}}{\left(1-v^{\prime 2}\right)^{3 / 2}}=(N-1) \frac{v^{\prime}}{r \sqrt{1-v^{\prime 2}}}+N \lambda\left(\frac{f^{\prime}\left(\varphi^{-1}(v)\right)}{\sqrt{1-v^{\prime 2}}}-f\left(\varphi^{-1}(v)\right) H\left(\varphi^{-1}(v), r\right)\right) \text {. }
$$

Note that the above equation is singular at $r=0$, which raises some essential difficulties. To remove this singularity, we consider the following approximation problem

$$
\left\{\begin{array}{l}
-\frac{v^{\prime \prime}}{\left(1-v^{\prime 2}\right)^{3 / 2}}=(N-1) \frac{v^{\prime}}{(r+\varepsilon) \sqrt{1-v^{\prime 2}}}+N \lambda\left(\frac{f^{\prime}\left(\varphi^{-1}(v)\right)}{\sqrt{1-v^{\prime 2}}}-f\left(\varphi^{-1}(v)\right) H\left(\varphi^{-1}(v), r\right)\right) \\
v^{\prime}(0)=v(R)=0
\end{array}\right.
$$

for any given $\varepsilon \in(0,1]$.

To study the bifurcation phenomenon of problem (2.1), we consider the following auxiliary problem

$$
\left\{\begin{array}{l}
-\left((r+\varepsilon)^{N-1} \phi\left(v^{\prime}\right)\right)^{\prime}=(r+\varepsilon)^{N-1} g(r), r \in(0, R) \\
v^{\prime}(0)=v(R)=0
\end{array}\right.
$$

for any given $g \in Y$, where $Y$ denotes the Banach space of continuous functions on $[0, R]$ endowed with the uniform norm $\|\cdot\|_{\infty}$. Define the continuous linear operator $H: Y \longrightarrow$ $C^{1}[0, R]$ by

$$
H u(r)=(r+\varepsilon)^{1-N} \int_{0}^{r}(s+\varepsilon)^{N-1} u(s) d s
$$

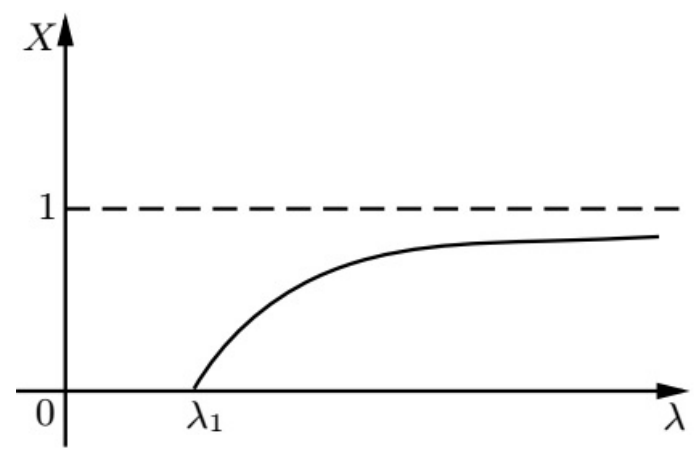

(a) $f_{0}+H_{0}=1$

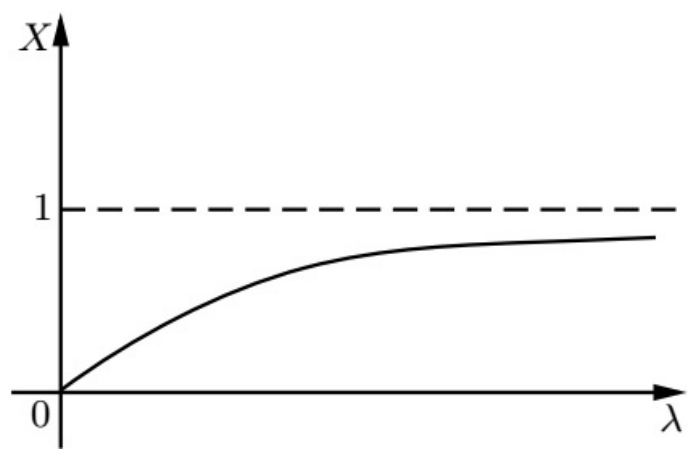

(b) $f_{0}+H_{0}=+\infty$

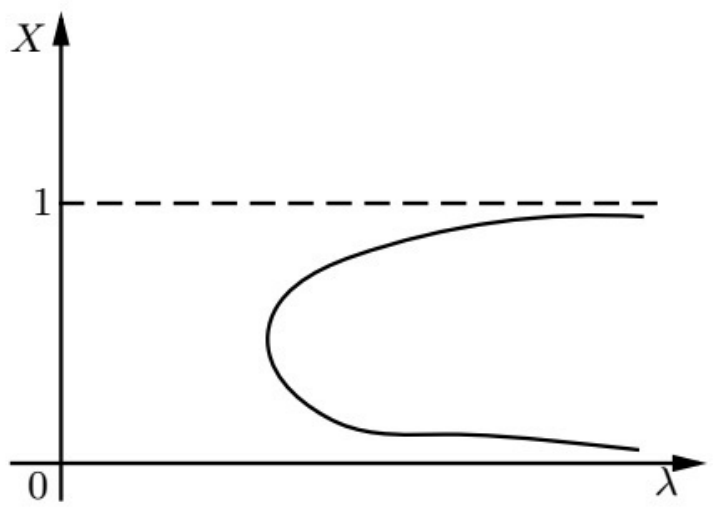

(c) $f_{0}+H_{0}=0$

Figure 1: Bifurcation diagrams of Theorem 1.1. 
for any $r \in[0, R]$.

Lemma 2.1. For each $g \in Y$, problem (2.2) has a unique solution given by

$$
v=\int_{r}^{R} \phi^{-1} \circ H(g) d s=\Psi_{\varepsilon}(g)
$$

for any $r \in[0, R]$.

Proof. Integrating the first equation of problem (2.2) from 0 to $r \in[0, R]$, we have that

$$
-\phi\left(v^{\prime}\right)=H(g) \text {. }
$$

Note that $\phi:(-1,1) \rightarrow \mathbb{R}$ is an increasing diffeomorphism satisfying $\phi(0)=0$. It follows that

$$
v^{\prime}=-\phi^{-1} \circ H(g) .
$$

Integrating the last equation from $R$ to $r$, in view of $v(R)=0$, we arrive at

$$
v=\int_{r}^{R} \phi^{-1} \circ H(g) d s
$$

which is the desired conclusion.

From Lemma 2.1, we can see that $v$ is a solution of problem (2.2) if and only if $v=\Psi_{\varepsilon}(g)$. Furthermore, we have the following compactness result.

Lemma 2.2. The operator $\Psi_{\varepsilon}: Y \longrightarrow \mathcal{B}_{1}(0)$ is continuous and sends bounded sets in $Y$ into relatively compact sets in $\mathcal{B}_{1}(0)$, where $\mathcal{B}_{1}(0)=\{u \in X:\|u\|<1\}$.

Proof. Noting the argument of Lemma 2.1, we have that

$$
\left|\phi\left(v^{\prime}\right)\right|=\left|(r+\varepsilon)^{1-N} \int_{0}^{r}(s+\varepsilon)^{N-1} g(s) d s\right| \leq R\|g\|_{\infty}:=M
$$

for any $r \in[0, R]$. It follows that

$$
\left|v^{\prime}\right| \leq \phi^{-1}(M)<1
$$

where $\phi^{-1}$ denotes the inverse function of $\phi$. So, $\Psi_{\varepsilon}$ maps $Y$ into $\mathcal{B}_{1}(0)$.

The continuity of $\Psi_{\varepsilon}$ is obvious. Similar to that of [19, Lemma 2.1], it suffices to prove that if $\left\{g_{n}\right\}$ is a bounded subsequence in $Y$ with $\left\|g_{n}\right\|_{\infty} \leq M$ for some positive constant $M$ and any $n \in \mathbb{N}$, then $v_{n}=\Psi_{\varepsilon}\left(g_{n}\right)$ contains a convergent subsequence in $\mathcal{B}_{1}(0)$. Clearly, one has that $\left\{H\left(g_{n}\right)\right\}$ is uniformly bounded. For any $r \in[0, R]$, by some simple calculations, we can show that

$$
\begin{aligned}
\left|\left(H\left(g_{n}\right)\right)^{\prime}(r)\right| & =\left|g_{n}(r)-(N-1) \frac{\int_{0}^{r}(s+\varepsilon)^{N-1} g_{n}(s) d s}{(r+\varepsilon)^{N}}\right| \\
& \leq M\left(1+(N-1) \frac{\int_{0}^{r}(s+\varepsilon)^{N-1} d s}{(r+\varepsilon)^{N}}\right) \\
& =M\left(1+\frac{(N-1)}{N} \frac{(r+\varepsilon)^{N}-\varepsilon^{N}}{(r+\varepsilon)^{N}}\right) \\
& \leq \frac{M(2 N-1)}{N} .
\end{aligned}
$$


For any $r, r^{\prime} \in[0, R]$, it follows from the above inequality and the Lagrange mean theorem that

$$
\left|\left(H\left(g_{n}\right)\right)^{\prime}(r)-\left(H\left(g_{n}\right)\right)^{\prime}\left(r^{\prime}\right)\right| \leq \frac{M(2 N-1)}{N}\left|r-r^{\prime}\right|
$$

So, the sequence $\left\{H\left(g_{n}\right)\right\}$ is also equi-continuous. By the Arzelà-Ascoli Theorem, there is a subsequence of $\left\{H\left(g_{n}\right)\right\}$, which we rename the same, which is convergent in $Y$. The limit of $\left\{H\left(g_{n}\right)\right\}$ in $Y$ is denoted by $g_{0}$. Then, it is easy to verify that $\phi^{-1}(s)=s / \sqrt{1+s^{2}}$. So, $\phi^{-1}: Y \longrightarrow Y$ is continuous. It follows that $v_{n}^{\prime}=-\phi^{-1}\left(H\left(g_{n}\right)\right)$ converges to $-\phi^{-1}\left(g_{0}\right):=v_{0}$ in $Y$. Reasoning as the first paragraph, we have that $\left\|v_{0}\right\|_{\infty}<1$. Therefore, we have that $v_{n}$ converges to $\int_{0}^{r} v_{0}(s) d s:=v$ in $\mathcal{B}_{1}(0)$.

We also need consider the following auxiliary problem

$$
\left\{\begin{array}{l}
-\left((r+\varepsilon)^{N-1} u^{\prime}\right)^{\prime}=(r+\varepsilon)^{N-1} h(r) \quad \text { in }(0, R), \\
u^{\prime}(0)=u(R)=0
\end{array}\right.
$$

for a given $h \in Y$. Analogously to that of Lemmas 2.1 and 2.2, we can show that problem (2.3) has a unique solution, which is denote by $\Phi_{\varepsilon}(h)$, and $\Phi_{\varepsilon}: Y \longrightarrow X$ is continuous, compact and linear.

Further, we consider the following problem with a parameter

$$
\left\{\begin{array}{l}
-\left((r+\varepsilon)^{N-1} \frac{v^{\prime}}{\sqrt{1-t^{2} v^{\prime 2}}}\right)^{\prime}=(r+\varepsilon)^{N-1} g(r), r \in(0, R), \\
v^{\prime}(0)=v(R)=0
\end{array}\right.
$$

for any $t \in(0,1]$ and any given $g \in Y$. Letting $w=t v$, problem (2.4) is equivalent to

$$
\left\{\begin{array}{l}
-\left((r+\varepsilon)^{N-1} \phi\left(w^{\prime}\right)\right)^{\prime}=t(r+\varepsilon)^{N-1} g(r), r \in(0, R), \\
w^{\prime}(0)=w(R)=0 .
\end{array}\right.
$$

By Lemma 2.1, problem (2.5) has a unique solution $w=\Psi_{\varepsilon}(t g)$. So $v=\Psi_{\varepsilon}(t g) / t$ is the unique solution of problem (2.4). For any $g \in Y$, define

$$
G_{\varepsilon}(t, g)= \begin{cases}\frac{\Psi_{\varepsilon}(t g)}{t} & \text { if } t \in(0,1] \\ \Phi_{\varepsilon}(g) & \text { if } t=0\end{cases}
$$

Then we can show that:

Lemma 2.3. $G_{\varepsilon}:[0,1] \times Y \longrightarrow X$ is completely continuous.

Proof. We first prove the continuity of $G_{\varepsilon}$. For any $g_{n}, g \in Y$ and $t_{n}, t \in[0,1]$ with $g_{n} \rightarrow g$ in $Y$ and $t_{n} \rightarrow t$ in $[0,1]$ as $n \rightarrow+\infty$, it is sufficient to show that $G_{\varepsilon}\left(t_{n}, g_{n}\right):=v_{n} \rightarrow$ $G_{\varepsilon}(t, g):=v$ in $X$.

If $t>0$, without loss of generality, we can assume that $t_{n}>0$ for any $n \in \mathbb{N}$. It follows from Lemma 2.2 that $v_{n} \rightarrow v$ in $X$ as $n \rightarrow+\infty$.

If $t=0$ and there exists a subsequence $t_{n_{i}}$ of $t_{n}$ such that $t_{n_{i}}=0$, then $v_{n_{i}}=$ $G_{\varepsilon}\left(t_{n_{i}}, g_{n_{i}}\right)=\Phi_{\varepsilon}\left(g_{n_{i}}\right) \rightarrow \Phi_{\varepsilon}(g)=v$ in $X$ as $i \rightarrow+\infty$. So, next we assume that $t=0$ and $t_{n}>0$ for any $n \in \mathbb{N}$. From Lemma 2.1 we know that problem (2.5) has only trivial solution when $t=0$. By Lemma 2.2, we have that $w_{n} \rightarrow 0$ in $X$ as $n \rightarrow+\infty$. 
Note that $v_{n}$ satisfies

$$
\left\{\begin{array}{l}
-\frac{v^{\prime \prime}}{\left(1-w_{n}^{\prime 2}\right)^{3 / 2}}-(N-1) \frac{v^{\prime}}{(r+\varepsilon) \sqrt{1-w_{n}^{\prime 2}}}=g_{n}(r), r \in(0, R), \\
v^{\prime}(0)=v(R)=0 .
\end{array}\right.
$$

It follows that there exists $N_{0}>0$ such that $\left\|v_{n}\right\|_{C^{2}[0, R]} \leq C$ for any $n \geq N_{0}$ and some positive constant $C$ which depends only on $g$ and $\varepsilon$. So, there exists $v \in X$ and a subsequence $v_{n_{k}}$ of $v_{n}$ such that $v_{n_{k}} \rightarrow v$ in $X$ as $k \rightarrow+\infty$. Note that

$$
v_{n_{k}}^{\prime \prime}=-(N-1) \frac{v_{n_{k}}^{\prime}\left(1-w_{n_{k}}^{\prime 2}\right)}{r+\varepsilon}-\left(1-w_{n_{k}}^{\prime 2}\right)^{3 / 2} g_{n_{k}}(r), r \in(0, R) .
$$

Integrating the above equation from 0 to $r \in(0, R)$, we get that

$$
v_{n_{k}}^{\prime}(r)=\int_{0}^{r}\left(-(N-1) \frac{v_{n_{k}}^{\prime}\left(1-w_{n_{k}}^{\prime 2}\right)}{s+\varepsilon}-\left(1-w_{n_{k}}^{\prime 2}\right)^{3 / 2} g_{n_{k}}(s)\right) d s .
$$

Noting $w_{n_{k}} \rightarrow 0$ in $X$ as $k \rightarrow+\infty$, by the Lebesgue Dominated Convergence Theorem, we have that

$$
v^{\prime}(r)=\int_{0}^{r}\left(-(N-1) \frac{v^{\prime}}{s+\varepsilon}-g(s)\right) d s .
$$

It follows that

$$
\left\{\begin{array}{l}
-\left((r+\varepsilon)^{N-1} v^{\prime}\right)^{\prime}=(r+\varepsilon)^{N-1} g(r), r \in(0, R), \\
v^{\prime}(0)=v(R)=0 .
\end{array}\right.
$$

Hence, one has that $v=\Phi_{\varepsilon}(g)=G_{\varepsilon}(0, g)$. We claim that $v_{n} \rightarrow v$ in $X$. Otherwise, there would exist a subsequence $\left\{v_{m_{j}}\right\}$ of $\left\{v_{n}\right\}$ in $X$ and $\epsilon_{0}>0$ such that for any $j \in \mathbb{N}$, we have $\left\|v_{m_{j}}-v\right\| \geq \epsilon_{0}$. But reasoning as above, $\left\{v_{m_{j}}\right\}$ would contain a further subsequence $v_{m_{j_{l}}} \rightarrow v$ in $X$ as $l \rightarrow+\infty$, which contradicts $\left\|v_{m_{j_{l}}}-v\right\| \geq \epsilon_{0}$. Therefore, $v_{n} \rightarrow v$ in $X$.

Next, we show the compactness of $G_{\varepsilon}$. Clearly, $G_{\varepsilon}(t, \cdot)$ is compact for any fixed $t \in$ $[0,1]$. We claim that the continuity of $G_{\varepsilon}$ with respect to $t$ at any $t_{0} \in[0,1]$ is uniform for $g \in Y$. That is to say, for any $\epsilon>0$ and $g \in Y$, there exists $\delta=\delta\left(\epsilon, t_{0}\right)>0$ such that $\left\|G_{\varepsilon}(t, g)-G_{\varepsilon}\left(t_{0}, g\right)\right\|<\epsilon$ when $\left|t-t_{0}\right|<\delta$ with $t \in[0,1]$. Suppose, by contradiction, that there exist $\epsilon_{0}>0, g_{0} \in Y$ such that for any $n \in \mathbb{N}$, existing $t_{n} \in[0,1]$ with $\left|t_{n}-t_{0}\right|<1 / n$ such that

$$
\left\|G_{\varepsilon}\left(t_{n}, g_{0}\right)-G_{\varepsilon}\left(t_{0}, g_{0}\right)\right\| \geq \epsilon_{0}
$$

Clearly, up to a subsequence, we have $t_{n} \rightarrow t_{0} \in[0,1]$ as $n \rightarrow+\infty$. Letting $n \rightarrow+\infty$ in (2.6), in view of the continuity of $G_{\varepsilon}$, we have that

$$
0=\lim _{n \rightarrow+\infty}\left\|G_{\varepsilon}\left(t_{n}, g_{0}\right)-G_{\varepsilon}\left(t_{0}, g_{0}\right)\right\| \geq \epsilon_{0}
$$

which is a contradiction.

For any $\left(t_{n}, g_{n}\right) \in[0,1] \times Y$ with $\left\{g_{n}\right\}$ is bounded in $Y$ for any $n \in \mathbb{N}$, it suffices to show that $\left\{G_{\varepsilon}\left(t_{n}, g_{n}\right)\right\}$ possesses a convergent subsequence. Without loss of generality, we assume that $t_{n} \rightarrow t_{0} \in[0,1]$. We have known that $\left\{G_{\varepsilon}\left(t_{1}, g_{n}\right)\right\}$ has a convergent subsequence. So, there exists a subsequence $\left\{g_{n}^{(1)}\right\}$ of $\left\{g_{n}\right\}$ such that the diameter of $\left\{G_{\varepsilon}\left(t_{1}, g_{n}^{(1)}\right)\right\}$ less than 1. Analogously, there exists $\left\{g_{n}^{(2)}\right\} \subseteq\left\{g_{n}^{(1)}\right\}$ such that the diameter 
of $\left\{G_{\varepsilon}\left(t_{2}, g_{n}^{(2)}\right)\right\}$ less than $1 / 2$. In general, there exists $\left\{g_{n}^{(k)}\right\} \subseteq\left\{g_{n}^{(k-1)}\right\}$ such that the diameter of $\left\{G_{\varepsilon}\left(t_{k}, g_{n}^{(k)}\right)\right\}$ less than $1 / k, k \geq 3$.

Next, we show that $\left\{G_{\varepsilon}\left(t_{n}, g_{n}^{(n)}\right)\right\}$ must be convergent. We have shown that, for any $\epsilon>0$ and $g \in Y$, there exists $\delta=\delta\left(\epsilon, t_{0}\right)>0$ such that $\left\|G_{\varepsilon}(t, g)-G_{\varepsilon}\left(t_{0}, g\right)\right\|<\epsilon / 3$ when $\left|t-t_{0}\right|<\delta$ with $t \in[0,1]$. Take $N_{1}>3 / \epsilon$ such that $\left|t_{n}-t_{0}\right|<\delta$ for any $n>N_{1}$. Consequently, when $m>n>N_{1}$, we have that

$$
\begin{aligned}
\left\|G_{\varepsilon}\left(t_{m}, g_{m}^{(m)}\right)-G_{\varepsilon}\left(t_{n}, g_{n}^{(n)}\right)\right\|< & \left\|G_{\varepsilon}\left(t_{m}, g_{m}^{(m)}\right)-G_{\varepsilon}\left(t_{0}, g_{m}^{(m)}\right)\right\| \\
& +\left\|G_{\varepsilon}\left(t_{0}, g_{m}^{(m)}\right)-G_{\varepsilon}\left(t_{n}, g_{m}^{(m)}\right)\right\| \\
& +\left\|G_{\varepsilon}\left(t_{n}, g_{m}^{(m)}\right)-G_{\varepsilon}\left(t_{n}, g_{n}^{(n)}\right)\right\| \\
< & \frac{\epsilon}{3}+\frac{\epsilon}{3}+\frac{1}{n}<\epsilon .
\end{aligned}
$$

It follows that $\left\{G_{\varepsilon}\left(t_{n}, g_{n}^{(n)}\right)\right\}$ is the Cauchy sequence. Thus, one has that $G_{\varepsilon}\left(t_{n}, g_{n}^{(n)}\right) \rightarrow v_{0}$ for some $v_{0} \in X$.

Finally, we prove that $G_{\varepsilon}\left(t_{n}^{(n)}, g_{n}^{(n)}\right) \rightarrow v_{0}$ as $n \rightarrow+\infty$. Clearly, there exists an $N_{2}>0$ such that $\left|t_{n}-t_{0}\right|<\delta,\left|t_{n}^{(n)}-t_{0}\right|<\delta$ and $\left\|G_{\varepsilon}\left(t_{n}, g_{n}^{(n)}\right)-v_{0}\right\|<\epsilon / 3$ any $n>N_{2}$. Hence, when $n>N_{2}$, we obtain that

$$
\begin{aligned}
\left\|G_{\varepsilon}\left(t_{n}^{(n)}, g_{n}^{(n)}\right)-v_{0}\right\|< & \left\|G_{\varepsilon}\left(t_{n}^{(n)}, g_{n}^{(n)}\right)-G_{\varepsilon}\left(t_{0}, g_{n}^{(n)}\right)\right\| \\
& +\left\|G_{\varepsilon}\left(t_{0}, g_{n}^{(n)}\right)-G_{\varepsilon}\left(t_{n}, g_{n}^{(n)}\right)\right\| \\
& +\left\|G_{\varepsilon}\left(t_{n}, g_{n}^{(n)}\right)-v_{0}\right\| \\
& <\frac{\epsilon}{3}+\frac{\epsilon}{3}+\frac{\epsilon}{3}<\epsilon .
\end{aligned}
$$

Therefore, we obtain that $G_{\varepsilon}\left(t_{n}^{(n)}, g_{n}^{(n)}\right) \rightarrow v_{0}$ in $X$ as $n \rightarrow+\infty$.

Let $\lambda_{1}(\varepsilon)$ be the first eigenvalue of

$$
\left\{\begin{array}{l}
-\left((r+\varepsilon)^{N-1} v^{\prime}\right)^{\prime}=\lambda(r+\varepsilon)^{N-1} v, r \in(0, R), \\
v^{\prime}(0)=v(R)=0
\end{array}\right.
$$

It is well known that $\lambda_{1}(\varepsilon)$ is simple, isolated and the associated eigenfunctions have one sign in $[0, R)$ (see $[17,31])$.

For any fixed $\lambda$, consider the following problem

$$
\left\{\begin{array}{l}
-\left((r+\varepsilon)^{N-1} \phi\left(v^{\prime}\right)\right)^{\prime}=\lambda(r+\varepsilon)^{N-1} v, r \in(0, R) \\
v^{\prime}(0)=v(R)=0
\end{array}\right.
$$

Obviously, problem (2.8) is equivalent to the operator equation $v=\Psi_{\varepsilon}(\lambda v):=\Psi_{\varepsilon, \lambda}(v)$. By Lemma 2.2 , we see that $\Psi_{\varepsilon, \lambda}: \mathcal{B}_{1}(0) \rightarrow \mathcal{B}_{1}(0)$ is complete continuous. Furthermore, by Lemma 2.3, we can obtain the following topological degree jumping result.

Lemma 2.4. For any $r \in(0,1)$, one has that

$$
\operatorname{deg}\left(I-\Psi_{\varepsilon, \lambda}, \mathcal{B}_{r}(0), 0\right)= \begin{cases}1 & \text { if } \lambda \in\left(0, \lambda_{1}\right) \\ -1 & \text { if } \lambda \in\left(\lambda_{1}, \lambda_{1}+\delta\right)\end{cases}
$$


for some $\delta>0$, where $\mathcal{B}_{r}(0)=\{u \in X:\|u\|<r\}$.

Proof. Since $\lambda_{1}(\varepsilon)$ is isolated, there exists $\delta=\delta(\varepsilon)>0$ such that problem (2.7) does not have eigenvalue in $\left(\lambda_{1}(\varepsilon), \lambda_{1}(\varepsilon)+\delta\right)$. We claim that the Leray-Schauder degree

$$
\operatorname{deg}\left(I-G_{\varepsilon}(t, \lambda \cdot), \mathcal{B}_{r}(0), 0\right)
$$

is well defined for any $\lambda \in\left(0, \lambda_{1}(\varepsilon)+\delta\right) \backslash\left\{\lambda_{1}(\varepsilon)\right\}$ and $t \in[0,1]$. It is clear for $t=0$. So, in view of Lemma 2.3, it is sufficient to show that $v=G_{\varepsilon}(t, \lambda v)$ has no solution with $\|v\|=r$ for $r$ sufficiently small and any $t \in(0,1]$. Otherwise, there exists a sequence $\left\{v_{n}\right\}$ such that $v_{n}=\Psi_{\varepsilon, \lambda}\left(t v_{n}\right) / t$ and $\left\|v_{n}\right\| \rightarrow 0$ as $n \rightarrow+\infty$. Letting $\widetilde{w}_{n}=v_{n} /\left\|v_{n}\right\|$, one has that $\widetilde{w}_{n}$ satisfies

$$
\left\{\begin{array}{l}
-\frac{\widetilde{w}_{n}^{\prime \prime}}{\left(1-w_{n}^{\prime 2}\right)^{3 / 2}}-(N-1) \frac{\widetilde{w}_{n}^{\prime}}{(r+\varepsilon) \sqrt{1-w_{n}^{\prime 2}}}=\lambda \widetilde{w}_{n}, r \in(0, R), \\
\widetilde{w}_{n}^{\prime}(0)=\widetilde{w}_{n}(R)=0 .
\end{array}\right.
$$

Similar to Lemma 2.3, we can show that, for some convenient subsequence, $\widetilde{w}_{n} \rightarrow \widetilde{w}$ as $n \rightarrow+\infty$ and $\widetilde{w}$ verifies problem $(2.7)$ with $\|\widetilde{w}\|=1$. This implies that $\lambda$ is an eigenvalue of problem (2.7), a contradiction.

Now, from the invariance of the degree under homotopies, we obtain that

$$
\begin{aligned}
\operatorname{deg}\left(I-\Psi_{\varepsilon, \lambda}, \mathcal{B}_{r}(0), 0\right) & =\operatorname{deg}\left(I-G_{\varepsilon}(1, \lambda \cdot), \mathcal{B}_{r}(0), 0\right)=\operatorname{deg}\left(I-G_{\varepsilon}(0, \lambda \cdot), \mathcal{B}_{r}(0), 0\right) \\
& =\operatorname{deg}\left(I-\lambda \Phi_{\varepsilon}, \mathcal{B}_{r}(0), 0\right)
\end{aligned}
$$

Since $\Phi_{\varepsilon}$ is compact and linear, by Theorem 8.10 of [13], we obtain that

$$
\operatorname{deg}\left(I-\lambda \Phi_{\varepsilon}, \mathcal{B}_{r}(0), 0\right)= \begin{cases}1 & \text { if } \lambda \in\left(0, \lambda_{1}(\varepsilon)\right) \\ -1 & \text { if } \lambda \in\left(\lambda_{1}(\varepsilon), \lambda_{1}(\varepsilon)+\delta\right) .\end{cases}
$$

Therefore, we have that

$$
\operatorname{deg}\left(I-\Psi_{\varepsilon, \lambda}, \mathcal{B}_{r}(0), 0\right)= \begin{cases}1 & \text { if } \lambda \in\left(0, \lambda_{1}(\varepsilon)\right) \\ -1 & \text { if } \lambda \in\left(\lambda_{1}(\varepsilon), \lambda_{1}(\varepsilon)+\delta\right) .\end{cases}
$$

This completes the proof.

Graphs which are solutions of problem (2.1) are spacelike on the open ball $B_{R}(0)$. The following lemma ensures a priori that each possible solution $v$ of problem (2.1) is spacelike on the boundary of $B_{R}(0)$, too.

Lemma 2.5. Let $v$ be any solution of problem (2.1). Then $\left|v^{\prime}\right|<1$ on $[0, R]$.

Proof. It is enough to show $\left|v^{\prime}(R)\right|<1$. Suppose, by contradiction, that there exists $\left\{r_{k}\right\} \subset(0, R)$ such that

$$
\lim _{k \rightarrow+\infty} r_{k}=R, \lim _{k \rightarrow+\infty}\left|v^{\prime}\left(r_{k}\right)\right|=\left|v^{\prime}(R)\right|=1 \text { and } \lim _{k \rightarrow+\infty}\left|\phi\left(v^{\prime}\right)\left(r_{k}\right)\right|=+\infty .
$$

By some elementary calculations, we have that

$$
\frac{\left((r+\varepsilon)^{N-1} \phi\left(v^{\prime}\right)\right)^{\prime}}{(r+\varepsilon)^{N-1} \phi\left(v^{\prime}\right)}=N \lambda\left(\frac{f\left(\varphi^{-1}(v)\right) H\left(\varphi^{-1}(v), r\right)}{\phi\left(v^{\prime}\right)}-\frac{f^{\prime}\left(\varphi^{-1}(v)\right)}{v^{\prime}}\right) .
$$


Clearly, there exists $\bar{r} \in(0, R)$ such that $\left|v^{\prime}\right|>1 / 2$ for all $r \in(\bar{r}, R)$. Integrating the above equality from $\bar{r}$ to $r_{k}$, we obtain that

$$
\begin{aligned}
\log \left|\left(r_{k}+\varepsilon\right)^{N-1} \phi\left(v^{\prime}\left(r_{k}\right)\right)\right| & -\log \left|(\bar{r}+\varepsilon)^{N-1} \phi\left(v^{\prime}(\bar{r})\right)\right| \\
& =N \lambda \int_{\bar{r}}^{r_{k}}\left(\frac{f\left(\varphi^{-1}(v)\right) H\left(r, \varphi^{-1}(v)\right)}{\phi\left(v^{\prime}\right)}-\frac{f^{\prime}\left(\varphi^{-1}(v)\right)}{v^{\prime}}\right) d r .
\end{aligned}
$$

Letting $k \rightarrow+\infty$, we can see that the left member tends to infinity while the right one is bounded, which is a contradiction.

Next, we show a Rabinowitz's type global bifurcation result for fully nonlinear operator equation which may not be globally defined. Let $E$ be a real Banach space with the norm $\|\cdot\|, \mathcal{O}$ be an open subset of $\mathbb{R} \times E$ and $\operatorname{pr}_{E}(\mathcal{O})$ be the projection of $\mathcal{O}$ on $E$. Consider the following fully operator equation

$$
u=F(\lambda, u),
$$

where $F: \mathcal{O} \longrightarrow \operatorname{pr}_{E}(\mathcal{O})$ is completely continuous with $F(\lambda, 0)=0$ for any $\lambda \in \operatorname{pr}_{\mathbb{R}}(\mathcal{O})$ with $\operatorname{pr}_{\mathbb{R}}(\mathcal{O})$ being the projection of $\mathcal{O}$ on $\mathbb{R}$.

Proposition 2.1. Let $a, b \in \operatorname{pr}_{\mathbb{R}}(\mathcal{O})$ with $a<b$, such that $u=0$ is an isolated solution of (2.9) for $\lambda=a$ and $\lambda=b$, where $a, b$ are not bifurcation points, furthermore assume that

$$
\operatorname{deg}\left(I-F(a, \cdot), \mathfrak{B}_{r}(0), 0\right) \neq \operatorname{deg}\left(I-F(b, \cdot), \mathfrak{B}_{r}(0), 0\right),
$$

where $\mathfrak{B}_{r}(0)=\left\{u \in \operatorname{pr}_{E}(\mathcal{O}):\|u\|<r\right\}$ is an isolating neighborhood of the trivial solution. Let

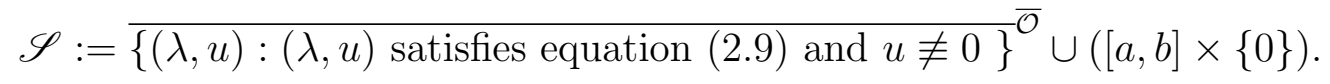

Then $\mathscr{S}$ possesses a maximal subcontinuum $\mathscr{C} \subset \overline{\mathcal{O}}$ such that $[a, b] \times\{0\} \subset \mathscr{C}$ and one of the following three properties is satisfied by $\mathscr{C}$ :

(i) $\mathscr{C}$ is unbounded in $\mathcal{O}$,

(ii) meets $\partial \mathcal{O}$,

(iii) meets $(\bar{\mu}, 0)$, where $\bar{\mu} \in \operatorname{pr}_{\mathbb{R}}(\mathcal{O}) \backslash[a, b]$.

Proof. Define

$$
\Gamma=\left\{\Omega \subseteq \mathcal{O}: \Omega=\Omega_{0} \cup \Omega_{\infty}\right\},
$$

where $\Omega_{0}=\mathfrak{B}_{r}(0) \times[a, b]$ and $\Omega_{\infty}$ is a bounded open subset of $\mathcal{O} \backslash\left(\operatorname{pr}_{\mathbb{R}}(\mathcal{O}) \times\{0\}\right)$. We claim that equation (2.9) has a nontrivial solution $(\lambda, u) \in \partial \Omega$ for any $\Omega \in \Gamma$. Let $f=I-F$, $K=f^{-1}(0) \cap \bar{\Omega}, A=[a, b] \times\{0\}$ and $B=f^{-1}(0) \cap\left(\partial \Omega \backslash\left(\{a\} \times \mathfrak{B}_{r}(0) \cup\{b\} \times \mathfrak{B}_{r}(0)\right)\right)$. Clearly, $K$ can be regarded as a compact metric space, and $A$ is a compact subset of $K$. If $B \neq \emptyset$, obviously, $B$ is also a compact subset of $K$. Apply the Whyburn's lemma [32, Chap. I, Statement (9.3)], we have that either there exists a continuum in $K$ connecting $A$ to $B$ or else, there is a separation $K_{A}, K_{B}$ of $K$ with $A \subset K_{A}, B \subset K_{B}$. If the former case occurs, the claim is verified. If the latter holds, we can find open sets $U$ and $V$ in $\mathcal{O}$ such that $K_{A} \subset U$ and $K_{B} \subset V$ with $U \cap V=\emptyset$. Set $\Omega^{*}=\Omega \cap(U \cup V)$. Then it is not difficult to see that $\Omega^{*} \in \Gamma$. It follows that there are no nontrivial solutions of equation (2.9) which belong to $\partial \Omega^{*}$. Then, by the generalized homotopy [28, Theorem 4.1] and the excision principle of Leray-Schauder degree, one has that

$$
\operatorname{deg}\left(I-F(a, \cdot), \mathfrak{B}_{r}(0), 0\right)=\operatorname{deg}\left(I-F(b, \cdot), \mathfrak{B}_{r}(0), 0\right),
$$


contradicting (2.10). If $B=\emptyset$, we can find an open set $U$ in $\mathcal{O}$ such that $K_{A} \subset U$. Then reasoning as the above, we still can get a contradiction.

By contradiction, we assume that $\mathscr{C}$ not satisfies any the above three properties. Then, there exists $\Omega \in \Gamma$ such $\partial \Omega$ not contains nontrivial solution of equation (2.9), which contracts the above claim.

If $\mathcal{O}=\mathbb{R} \times E$, Proposition 2.1 is just Theorem 4.12 of [28]. So, Proposition 2.1 can be seen the local version of Theorem 4.12 of [28].

Theorem 2.1. Assume that $f^{\prime}(t) \geq 0$ for any $t \in I_{f}(R)$ and $f^{\prime}(t)>f(t) H(t, r)$ for any $r \in[0, R], t \in I_{f}(R) \backslash\{0\}$, and there exist $f_{0} \in[0,+\infty], H_{0} \in \mathbb{R} \cup\{-\infty,+\infty\}$ with $f_{0}+H_{0} \in[0,+\infty]$ such that

$$
\lim _{t \rightarrow 0^{+}} \frac{N f^{\prime}(t)}{\varphi(t)}=f_{0}, \lim _{t \rightarrow 0^{+}} \frac{N f(t) H(t, r)}{\varphi(t)}=-H_{0}
$$

uniformly for $r \in(0, R)$. Then,

(a) if $f_{0}+H_{0}=1$, there is an unbounded component $\mathscr{C}_{\varepsilon}$ of the set of positive solutions of problem (2.1) bifurcating from $\left(\lambda_{1}(\varepsilon), 0\right)$ such that $\mathscr{C}_{\varepsilon} \subseteq\left(\left(\left(\mathbb{R}_{+} \backslash\{0\}\right) \times X\right) \cup\left\{\left(\lambda_{1}(\varepsilon), 0\right)\right\}\right)$, $\left(\lambda_{1}(\varepsilon),+\infty\right) \subseteq p r_{\mathbb{R}}\left(\mathscr{C}_{\varepsilon}\right),\left\|v_{\varepsilon, \lambda}\right\|<1$ and $\lim _{\lambda \rightarrow+\infty}\left\|v_{\varepsilon, \lambda}\right\|=1$ for $\left(\lambda, v_{\varepsilon, \lambda}\right) \in \mathscr{C}_{\varepsilon} \backslash\left\{\left(\lambda_{1}(\varepsilon), 0\right)\right\}$,

(b) if $f_{0}+H_{0}=+\infty$, there is an unbounded component $\mathscr{C}_{\varepsilon}$ of the set of positive solutions of problem (2.1) bifurcating from $(0,0)$ and joining to $(+\infty, 1)$ such that $\mathscr{C}_{\varepsilon} \subseteq$ $\left(\left(\left(\mathbb{R}_{+} \backslash\{0\}\right) \times X\right) \cup\{(0,0)\}\right)$ and $\left\|v_{\varepsilon, \lambda}\right\|<1$ for any $\left(\lambda, v_{\varepsilon, \lambda}\right) \in \mathscr{C}_{\varepsilon} \backslash\{(0,0)\}$ with $\lambda<+\infty$,

(c) if $f_{0}+H_{0}=0$, there is an unbounded component $\mathscr{C}_{\varepsilon}$ of the set of positive solutions of problem (2.1) in $\mathbb{R}_{+} \times X$ which joins $(+\infty, 1)$ to $(+\infty, 0)$ and $\left\|v_{\varepsilon, \lambda}\right\|<1$ for any $\left(\lambda, v_{\varepsilon, \lambda}\right) \in \mathscr{C}_{\varepsilon} \backslash\{(0,0)\}$ with $\lambda<+\infty$.

Proof. (a) Let $\xi(t, r)=N f(t) H(t, r)+H_{0} \varphi(t)$. Then, we have that

$$
\lim _{t \rightarrow 0^{+}} \frac{\xi(t, r)}{\varphi(t)}=0
$$

Let $\eta(s, r)=\xi\left(\varphi^{-1}(s), r\right)$. It is easy to see that

$$
\lim _{s \rightarrow 0^{+}} \frac{\eta(s, r)}{s}=0
$$

uniformly for $r \in(0, R)$. Letting $\zeta(s)=N f^{\prime}\left(\varphi^{-1}(s)\right)-f_{0} s$, one has that

$$
\lim _{s \rightarrow 0^{+}} \frac{\zeta(s)}{s}=0 .
$$

Consider

$$
\left\{\begin{array}{l}
, r-\left((r+\varepsilon)^{N-1} \phi\left(v^{\prime}\right)\right)^{\prime}=\lambda(r+\varepsilon)^{N-1}\left(\frac{f_{0} v+\zeta(v)}{\sqrt{1-v^{\prime 2}}}-\left(\eta(v)-H_{0} v\right)\right), \\
v^{\prime}(0)=v(R)=0
\end{array}\right.
$$

as a bifurcation problem from the trivial solution axis.

For each $\varepsilon>0$, define $F_{\lambda}(s, v):[0,1] \times \mathcal{B}_{1}(0) \rightarrow Y$

$$
F_{\lambda}(s, v)=\lambda\left(\frac{f_{0} v+s \zeta(v)}{\sqrt{1-s v^{\prime 2}}}+H_{0} v-s \eta(v, r)\right) .
$$


Then, it is easy to see that $F_{\lambda}$ is continuous and takes bounded sets into bounded sets. Consider the following problem

$$
\left\{\begin{array}{l}
-\left((r+\varepsilon)^{N-1} \phi_{\varepsilon}\left(v^{\prime}\right)\right)^{\prime}=(r+\varepsilon)^{N-1} F_{\lambda}(s, v) \\
v^{\prime}(0)=v(R)=0
\end{array}\right.
$$

Then, problem (2.13) can be equivalently rewritten as

$$
v=\Psi_{\varepsilon}\left(F_{\lambda}(s, v)\right):=T_{\varepsilon, \lambda}(s, v) .
$$

By Lemma $2.2, T_{\varepsilon, \lambda}:[0,1] \times \mathcal{B}_{1}(0) \rightarrow \mathcal{B}_{1}(0)$ is completely continuous. In particular, $H_{\varepsilon, \lambda}:=$ $T_{\varepsilon, \lambda}(1, \cdot): \mathcal{B}_{1}(0) \rightarrow \mathcal{B}_{1}(0)$ is completely continuous.

Let

$$
\widetilde{\eta}(u, r)=\max _{0 \leq s \leq u}|\xi(s, r)| \text { for any } r \in(0, R) .
$$

Then, we have that $\widetilde{\eta}$ is nondecreasing with respect to $u$ and

$$
\lim _{u \rightarrow 0^{+}} \frac{\widetilde{\eta}(u, r)}{u}=0
$$

Further, it follows from (2.14) that

$$
\left|\frac{\eta(v, r)}{\|v\|}\right| \leq \frac{\widetilde{\eta}(v, r)}{\|v\|} \leq \frac{\widetilde{\eta}\left(\|v\|_{\infty}, r\right)}{\|v\|} \leq R \frac{\widetilde{\eta}(R\|v\|, r)}{R\|v\|} \rightarrow 0 \text { as }\|v\| \rightarrow 0
$$

uniformly in $r \in(0, R)$. In a similar manner, we can show that

$$
\left|\frac{\zeta(v)}{\|v\|}\right| \rightarrow 0 \text { as }\|v\| \rightarrow 0
$$

We claim that the Leray-Schauder degree $\operatorname{deg}\left(I-T_{\varepsilon, \lambda}(s, \cdot), \mathcal{B}_{r}(0), 0\right)$ is well defined for $\lambda \in\left(0, \lambda_{1}(\varepsilon)+\delta\right) \backslash\left\{\lambda_{1}(\varepsilon)\right\}$ and $r$ small enough. Suppose, on the contrary, that there exists a sequence $\left\{v_{n}\right\}$ such that $v_{n}=T_{\varepsilon, \lambda}\left(s, v_{n}\right)$ and $\left\|v_{n}\right\| \rightarrow 0$ as $n \rightarrow+\infty$. Letting $\widehat{w}_{n}=v_{n} /\left\|v_{n}\right\|$, we have that $\widehat{w}_{n}$ satisfies

$$
\left\{\begin{array}{l}
-\frac{\widehat{w}_{n}^{\prime \prime}}{\left(1-v_{n}^{\prime 2}\right)^{3 / 2}}-(N-1) \frac{\widehat{w}_{n}^{\prime}}{(r+\varepsilon) \sqrt{1-v_{n}^{\prime 2}}}=\lambda\left(\frac{f_{0} \widehat{w}_{n}}{\sqrt{1-s v_{n}^{\prime 2}}}+\frac{s \zeta\left(v_{n}\right)}{\left\|v_{n}\right\| \sqrt{1-s v_{n}^{\prime 2}}}-s \frac{\eta\left(v_{n}, r\right)}{\left\|v_{n}\right\|}+H_{0} \widehat{w}_{n}\right) \\
\widehat{w}_{n}^{\prime}(0)=\widehat{w}_{n}(R)=0 .
\end{array}\right.
$$

Then, by (2.15), (2.16) and an argument similar to that of Lemma 2.3, we can show that $\widehat{w}_{n} \rightarrow \widehat{w}$ in $X$ as $n \rightarrow+\infty$ and

$$
\left\{\begin{array}{l}
-\left((r+\varepsilon)^{N-1} \widehat{w}^{\prime}\right)^{\prime}=\lambda(r+\varepsilon)^{N-1} \widehat{w}, \quad r \in(0, R), \\
\widehat{w}^{\prime}(0)=\widehat{w}(R)=0 .
\end{array}\right.
$$

Clearly, one has $\|\widehat{w}\|=1$. So, $\lambda$ is an eigenvalue of problem (2.7), which is absurd. Note that the above argument also shows that if $(\mu, 0)$ is a bifurcation point of nontrivial nonnegative solutions of problem (2.12), then $\mu$ is an eigenvalue of problem (2.7).

Now, by the invariance of the degree under homotopies, we obtain that

$$
\begin{aligned}
\operatorname{deg}\left(I-H_{\varepsilon, \lambda}, \mathcal{B}_{r}(0), 0\right) & =\operatorname{deg}\left(I-T_{\varepsilon, \lambda}(1, \cdot), \mathcal{B}_{r}(0), 0\right)=\operatorname{deg}\left(I-T_{\varepsilon, \lambda}(0, \cdot), \mathcal{B}_{r}(0), 0\right) \\
& =\operatorname{deg}\left(I-\Psi_{\varepsilon, \lambda}, \mathcal{B}_{r}(0), 0\right)
\end{aligned}
$$


Using Lemma 2.4, we have that

$$
\operatorname{deg}\left(I-H_{\varepsilon, \lambda}, \mathcal{B}_{r}(0), 0\right)= \begin{cases}1 & \text { if } \lambda \in\left(0, \lambda_{1}(\varepsilon)\right) \\ -1 & \text { if } \lambda \in\left(\lambda_{1}(\varepsilon), \lambda_{1}(\varepsilon)+\delta\right) .\end{cases}
$$

By Proposition 2.1 with $\mathcal{O}=\mathbb{R} \times \mathcal{B}_{1}(0)$, there exists a continuum $\mathscr{C}_{\varepsilon}$ of nontrivial solution of problem (2.12) bifurcating from $\left(\lambda_{1}(\varepsilon), 0\right)$ which satisfies one of the following three properties:

(i) $\mathscr{C}_{\varepsilon}$ is unbounded in $\mathcal{O}$,

(ii) meets $\partial \mathcal{O}$,

(iii) $\mathscr{C}_{\varepsilon} \cap\left(\mathbb{R} \backslash\left\{\lambda_{1}(\varepsilon)\right\} \times\{0\}\right) \neq \emptyset$.

By Lemma 2.5, we know that the second alternative is impossible. Since $(0,0)$ is the only solution of problem (2.1) for $\lambda=0$ and 0 is not an eigenvalue of problem (2.7), so $\mathscr{C}_{\varepsilon} \cap(\{0\} \times X)=\emptyset$. From the argument of Theorem 4.1 of [3], we know that $v_{\varepsilon}$ is nonnegative and decreasing for any $\left(\lambda, v_{\varepsilon}\right) \in \mathscr{C}_{\varepsilon}$.

We claim that $\mathscr{C}_{\varepsilon} \cap\left(\mathbb{R} \backslash\left\{\lambda_{1}(\varepsilon)\right\} \times\{0\}\right)=\emptyset$. Otherwise, there exists a nontrivial nonnegative solution sequence $\left(\lambda_{n}, v_{\varepsilon, n}\right) \in \mathscr{C}_{\varepsilon} \backslash\left\{\left(\lambda_{1}(\varepsilon), 0\right)\right\}$ such that $\lambda_{n} \rightarrow \mu$ and $v_{\varepsilon, n} \rightarrow 0$ as $n \rightarrow+\infty$. Let $w_{\varepsilon, n}:=v_{\varepsilon, n} /\left\|v_{\varepsilon, n}\right\|$, by (2.15), (2.16) and an argument like that of Lemma 2.3 , we can show that $w_{\varepsilon, n} \rightarrow w_{\varepsilon}$ as $n \rightarrow+\infty$ and $w_{\varepsilon}$ verifies problem $(2.7)$ with $\left\|w_{\varepsilon}\right\|=1$. It follows that $\mu=\lambda_{1}(\varepsilon)$, a contradiction.

Therefore, $\mathscr{C}_{\varepsilon}$ is unbounded in $(0,+\infty) \times \mathcal{B}_{1}(0)$ and $v_{\varepsilon}$ is nontrivial nonnegative for any $\left(\lambda, v_{\varepsilon}\right) \in \mathscr{C}_{\varepsilon} \backslash\left\{\left(\lambda_{1}(\varepsilon), 0\right)\right\}$. Furthermore, as that of Proposition 3.3 of [3], we can show that $v_{\varepsilon}$ is positive and strictly decreasing for any $\left(\lambda, v_{\varepsilon}\right) \in \mathscr{C}_{\varepsilon} \backslash\left\{\left(\lambda_{1}(\varepsilon), 0\right)\right\}$. The fact of $\left\|v_{\varepsilon}\right\|<1$ for any fixed $\left(\lambda, v_{\varepsilon}\right) \in \mathscr{C}_{\varepsilon}$ implies that the projection of $\mathscr{C}_{\varepsilon}$ on $\mathbb{R}_{+}$is unbounded.

Finally, we show the asymptotic behavior of $v_{\varepsilon, \lambda}$ as $\lambda \rightarrow+\infty$ for $\left(\lambda, v_{\varepsilon, \lambda}\right) \in \mathscr{C}_{\varepsilon} \backslash$ $\left\{\left(\lambda_{1}(\varepsilon), 0\right)\right\}$. Suppose, by contradiction, that there exist a constant $\delta>0$ and $\left(\lambda_{n}, v_{\varepsilon, n}\right) \in$ $\mathscr{C}_{\varepsilon} \backslash\left\{\left(\lambda_{1}(\varepsilon), 0\right)\right\}$ with $\lambda_{n} \rightarrow+\infty$ as $n \rightarrow+\infty$ such that $\left\|v_{\varepsilon, n}\right\|^{2} \leq 1-\delta^{2}$ for any $n \in \mathbb{N}$. Note that $\left(\lambda_{n}, v_{\varepsilon, n}\right)$ satisfies the following problem

$$
\left\{\begin{array}{l}
-\left((r+\varepsilon)^{N-1} \phi\left(v^{\prime}\right)\right)^{\prime}=\lambda N(r+\varepsilon)^{N-1} a(r) v, r \in(0, R), \\
v^{\prime}(0)=v(R)=0,
\end{array}\right.
$$

where

$$
a(r)=\frac{\frac{f^{\prime}\left(\varphi^{-1}(v)\right)}{\sqrt{1-v^{\prime 2}}}-f\left(\varphi^{-1}(v)\right) H\left(\varphi^{-1}(v), r\right)}{v(r)} .
$$

Since $f^{\prime}(t) \geq 0$, we have that

$$
a(r) \geq \frac{f^{\prime}\left(\varphi^{-1}(v)\right)-f\left(\varphi^{-1}(v)\right) H\left(\varphi^{-1}(v), r\right)}{v(r)} .
$$

The assumptions of $f_{0}+H_{0}=1$ and $f^{\prime}(t)>f(t) H(t, r)$ for any $r \in[0, R], t \in I_{f}(R) \backslash\{0\}$ imply that there exists a positive constant $\rho>0$ such that

$$
a(r) \geq \rho
$$

for any $r \in[0, R]$. 
Let $\varphi_{1}$ be a positive eigenfunction associated to $\lambda_{1}(\varepsilon)$. It is easy to see that $\varphi_{1}$ is decreasing in $[0, R]$. Multiplying the first equation of problem (2.17) by $\varphi_{1}$, and obtain after integrations by parts that

$$
\begin{aligned}
\frac{\lambda_{1}}{\delta} \int_{0}^{R}(r+\varepsilon)^{N-1} v_{\varepsilon, n} \varphi_{1} d r & =\frac{1}{\delta} \int_{0}^{R}(r+\varepsilon)^{N-1} v_{\varepsilon, n}^{\prime} \varphi_{1}^{\prime} d r \geq \int_{0}^{R}(r+\varepsilon)^{N-1} \frac{v_{\varepsilon, n}^{\prime} \varphi_{1}^{\prime}}{\sqrt{1-\left|v_{\varepsilon, n}^{\prime}\right|^{2}}} d r \\
& =\lambda_{n} N \int_{0}^{R}(r+\varepsilon)^{N-1} a(r) v_{\varepsilon, n} \varphi_{1} d r \\
& \geq \lambda_{n} N \rho \int_{0}^{R}(r+\varepsilon)^{N-1} v_{\varepsilon, n} \varphi_{1} d r .
\end{aligned}
$$

It follows that $\lambda_{n} \leq \lambda_{1}(\varepsilon) /(N \delta \rho)$, which is a contradiction.

(b) Let

$$
f(r, s, y)=\frac{f^{\prime}\left(\varphi^{-1}(s)\right)}{\sqrt{1-y^{2}}}-f\left(\varphi^{-1}(s)\right) H\left(\varphi^{-1}(s), r\right) .
$$

For any $n \in \mathbb{N}$, define

$$
f^{n}(r, s, y)= \begin{cases}n s, & s \in\left[0, \frac{1}{n}\right], \\ \left(f\left(r, \frac{2}{n}, y\right)-1\right) n s+2-f\left(r, \frac{2}{n}, y\right), & s \in\left(\frac{1}{n}, \frac{2}{n}\right), \\ f(r, s, y), & s \in\left[\frac{2}{n},+\infty\right) .\end{cases}
$$

Then, consider the following problem

$$
\left\{\begin{array}{l}
-\left((r+\varepsilon)^{N-1} \phi\left(v^{\prime}\right)\right)^{\prime}=\lambda N(r+\varepsilon)^{N-1} f^{n}\left(r, v, v^{\prime}\right), r \in(0, R) \\
v^{\prime}(0)=v(R)=0
\end{array}\right.
$$

By the conclusion of (a) and an argument similar to that of [12, Theorem 1.2], we can obtain the desired conclusion.

(c) For any $n \in \mathbb{N}$, define

$$
f_{n}(r, s, y)= \begin{cases}\frac{1}{n} s, & s \in\left[0, \frac{1}{n}\right] \\ \left(f\left(r, \frac{2}{n}, y\right)-\frac{1}{n^{2}}\right) n s+2 \frac{1}{n^{2}}-f\left(r, \frac{2}{n}, y\right), & s \in\left(\frac{1}{n}, \frac{2}{n}\right) \\ f(r, s, y), & s \in\left[\frac{2}{n},+\infty\right)\end{cases}
$$

and consider the following problem

$$
\left\{\begin{array}{l}
-\left((r+\varepsilon)^{N-1} \phi\left(v^{\prime}\right)\right)^{\prime}=\lambda N(r+\varepsilon)^{N-1} f_{n}\left(r, v, v^{\prime}\right), r \in(0, R) \\
v^{\prime}(0)=v(R)=0
\end{array}\right.
$$

Then, by an similar argument to that of Theorem 1.3 of [12] and the conclusion of (a), we can deduce the desired conclusion.

\section{Proof of Theorem 1.1}

Multiplying the first equation of problem $(2.1)$ by $\sqrt{1-v^{\prime 2}}$, we get that

$$
\left\{\begin{array}{l}
-\frac{v^{\prime \prime}}{1-v^{\prime 2}}=(N-1) \frac{v^{\prime}}{(r+\varepsilon)}+N \lambda\left(f^{\prime}\left(\varphi^{-1}(v)\right)-f\left(\varphi^{-1}(v)\right) H\left(\varphi^{-1}(v), r\right) \sqrt{1-v^{\prime 2}}\right) \\
v^{\prime}(0)=v(R)=0
\end{array}\right.
$$


Clearly, problem (3.1) is equivalent to problem (2.1). Define

$$
\psi(s)=-\frac{1}{2} \log \left(\frac{1+s}{1-s}\right), s \in(-1,1) .
$$

Then, $\psi$ is a decreasing diffeomorphism and satisfied $\psi(0)=0$. Following [3], we can rewrite the problem (3.1) as follows

$$
\left\{\begin{array}{l}
-\left(\frac{1}{2} \log \left(\frac{1+v^{\prime}}{1-v^{\prime}}\right)\right)^{\prime}=\frac{(N-1) v^{\prime}}{r+\varepsilon}+N \lambda\left(f^{\prime}\left(\varphi^{-1}(v)\right)-f\left(\varphi^{-1}(v)\right) H\left(\varphi^{-1}(v), r\right) \sqrt{1-v^{\prime 2}}\right), \\
v^{\prime}(0)=v(R)=0 .
\end{array}\right.
$$

From the argument of Theorem 4.1 of [3], for any solution $v_{\varepsilon}$ of problem (3.2), we know that, up to a subsequence, $v_{\varepsilon}$ is convergent in $X$ and the limit is a solution $v$ of problem (1.12). Therefore, in view of the definition of superior limit (see [32]), $\mathscr{C}:=\lim \sup _{\varepsilon \rightarrow 0^{+}} \mathscr{C}_{\varepsilon}$ is the solution set of problem (1.12).

To investigate the global structure of $\mathscr{C}$, we consider the eigenvalue problem (2.7) again.

Lemma 3.1. Up to a subsequence, one has that $\lambda_{1}(\varepsilon)$ converges to $\lambda_{1}$ as $\varepsilon \rightarrow 0^{+}$.

Proof. Firstly, we have the following Rayleigh quotient

$$
\lambda_{1}(\varepsilon)=\inf _{v \neq 0, v \in X} \frac{\int_{0}^{R}(r+\varepsilon)^{N-1} v^{\prime 2} d r}{\int_{0}^{R}(r+\varepsilon)^{N-1} v^{2} d r} .
$$

It follows that

$$
\lambda_{1}(\varepsilon) \leq \inf _{v \neq 0, v \in X} \frac{\int_{0}^{R}(r+1)^{N-1} v^{\prime 2} d r}{\int_{0}^{R} r^{N-1} v^{2} d r}:=\bar{\lambda}
$$

Then, $\bar{\lambda}$ is the first eigenvalue of

$$
\left\{\begin{array}{l}
-\left((r+1)^{N-1} u^{\prime}\right)^{\prime}=\lambda r^{N-1} u, r \in(0, R) \\
u^{\prime}(0)=u(R)=0
\end{array}\right.
$$

So, one has $\bar{\lambda}<+\infty$ (see $[17,31]$ ). Hence, $\lambda_{1}(\varepsilon)$ is bounded. Passing if necessary to a subsequence, we have $\lim _{\varepsilon \rightarrow 0^{+}} \lambda_{1}(\varepsilon)=\mu$.

Let $\varphi_{\varepsilon, 1}$ be the positive eigenfunction with $\left\|\varphi_{\varepsilon, 1}\right\|=1$ corresponding to $\lambda_{1}(\varepsilon)$. Passing to a subsequence if necessary, there exists $\varphi_{1} \in Y$ such that

$$
\lim _{\varepsilon \rightarrow 0^{+}}\left\|\varphi_{\varepsilon, 1}-\varphi_{1}\right\|_{\infty}=0
$$

Note that

$$
-\varphi_{\varepsilon, 1}^{\prime \prime}-(N-1) \frac{\varphi_{\varepsilon, 1}^{\prime}}{r+\varepsilon}=\lambda_{1}(\varepsilon) \varphi_{\varepsilon, 1}, r \in(0, R) .
$$

So, for any $a \in(0, R]$, we have that

$$
\left|\varphi_{\varepsilon, 1}^{\prime \prime}\right| \leq \frac{N-1}{a}+\lambda_{1}(\varepsilon) R, r \in[a, R] .
$$

Since $\lim _{\varepsilon \rightarrow 0^{+}} \lambda_{1}(\varepsilon)=\mu$, for any fixed $\sigma \in(0, \mu)$, there exists $\delta \in(0,1]$ such that $\lambda_{1}(\varepsilon)<$ $\mu+\sigma$ when $\varepsilon \in(0, \delta)$. So, when $\varepsilon \in(0, \delta)$, we have that

$$
\left|\varphi_{\varepsilon, 1}^{\prime \prime}\right| \leq \frac{N-1}{a}+R(\mu+\sigma), r \in[a, R] .
$$


It follows that $\varphi_{\varepsilon, 1}$ is uniformly bounded in $C^{2}[0, R]$. So, one has that $\varphi_{\varepsilon, 1}$ converges to $\varphi_{1}$ in $C^{1}[a, R]$.

Note that

$$
\left\{\begin{array}{l}
-\varphi_{\varepsilon, 1}^{\prime \prime}-(N-1) \frac{\varphi_{\varepsilon, 1}^{\prime}}{r+\varepsilon}=\lambda_{1}(\varepsilon) \varphi_{\varepsilon, 1}, r \in(0, R) \\
\varphi_{\varepsilon, 1}^{\prime}(0)=\varphi_{\varepsilon, 1}(R)=0 .
\end{array}\right.
$$

Integrating the first equation of problem (3.3) between $a$ and $R$, we infer that

$$
\varphi_{\varepsilon, 1}^{\prime}(a)-\varphi_{\varepsilon, 1}^{\prime}(R)=\int_{a}^{R}\left((N-1) \frac{\varphi_{\varepsilon, 1}^{\prime}}{r+\varepsilon}+\lambda_{1}(\varepsilon) \varphi_{\varepsilon, 1}\right) d r .
$$

By the Lebesgue Dominated Convergence Theorem, we get that

$$
\varphi_{1}^{\prime}(a)-\varphi_{1}^{\prime}(R)=\int_{a}^{R}\left((N-1) \frac{\varphi^{\prime}}{r}+\mu \varphi_{1}\right) d r .
$$

It follows that

$$
-\left(r^{N-1} \varphi_{1}^{\prime}\right)^{\prime}=\mu r^{N-1} \varphi_{1}, r \in(0, R) .
$$

Clearly, we have $\varphi_{1}(R)=0$.

Next, we show that $\varphi_{1}^{\prime}(0)=0$. Integrating the first equation of problem (3.3) from 0 and $R$, we have that

$$
(N-1) \int_{0}^{R} \frac{\varphi_{\varepsilon, 1}^{\prime}}{r+\varepsilon} d r=-\varphi_{\varepsilon, 1}^{\prime}(R)-\int_{0}^{R} \lambda_{1}(\varepsilon) \varphi_{\varepsilon, 1} d r .
$$

It follows that

$$
(N-1)\left|\int_{0}^{R} \frac{\varphi_{\varepsilon, 1}^{\prime}}{r+\varepsilon} d r\right| \leq 1+R^{2}(\mu+\sigma) .
$$

It is not difficult to verify that $\varphi_{\varepsilon, 1}$ is decreasing in $[0, R]$. It follows that

$$
(N-1) \int_{0}^{R} \frac{\left|\varphi_{\varepsilon, 1}^{\prime}\right|}{r+\varepsilon} d r=(N-1)\left|\int_{0}^{R} \frac{\varphi_{\varepsilon, 1}^{\prime}}{r+\varepsilon} d r\right| \leq 1+R^{2}(\mu+\sigma) .
$$

Hence, we have that

$$
\left\{-(N-1) \frac{\varphi_{\varepsilon, 1}^{\prime}}{r+\varepsilon}\right\}
$$

is a set of positive integrable functions. By the Fatou Lemma, we conclude that $-(N-1) \varphi_{1}^{\prime} / r$ is also integrable on $[0, R]$. For any $r \in(0, R]$, integrating equation (3.4) from $r$ to $R$, we get that

$$
\varphi_{1}^{\prime}(r)-\varphi_{1}^{\prime}(R)=\int_{r}^{R}\left((N-1) \frac{\varphi_{1}^{\prime}}{\tau}+\mu \varphi_{1}\right) d \tau
$$

Since $\varphi_{1} \in Y$, the limit of the right member exists when $r$ tends to 0 . So, we have the existence of $\lim _{r \rightarrow 0^{+}} \varphi_{1}^{\prime}(r)$. Then, by integrability of $\varphi_{1}^{\prime} / r$, we conclude that $\lim _{r \rightarrow 0^{+}} \varphi_{1}^{\prime}(r)=$ 0 .

Consequently, we obtain that $\varphi_{\varepsilon, 1}$ converges to $\varphi_{1}$ in $C^{1}[0, R]$ and

$$
\left\{\begin{array}{l}
-\left(r^{N-1} \varphi_{1}^{\prime}\right)^{\prime}=\mu r^{N-1} \varphi_{1}, r \in(0, R) \\
\varphi_{1}^{\prime}(0)=\varphi_{1}(R)=0
\end{array}\right.
$$


Since $\varphi_{\varepsilon, 1}$ is positive with $\left\|\varphi_{\varepsilon, 1}\right\|=1$, we have that $\varphi_{1}$ is nonnegative with $\left\|\varphi_{1}\right\|=1$. It follows that $\mu=\lambda_{1}$.

Now, we can present the proof of our main result. From now on, for simplicity, we take $\varepsilon=1 / n$ and rewrite $\mathscr{C}_{\varepsilon}$ by $\mathscr{C}_{n}$.

Proof of Theorem 1.1. (a) From Lemma 3.1 and Theorem 2.1, we know that $\left(\lambda_{1}, 0\right) \in$ $\liminf \operatorname{in}_{n \rightarrow+\infty} \mathscr{C}_{n}$. The compactness of $H_{\varepsilon, \lambda}$ implies that $\left(\cup_{n=1}^{+\infty} \mathscr{C}_{n}\right) \cap \mathcal{B}_{R}(0)$ is relatively compact. Theorem 2.1 of [11] implies that $\mathscr{C}$ is connected. Since $(+\infty, 1) \in \mathscr{C}_{n}$, we have $(+\infty, 1) \in \mathscr{C}$. So $\mathscr{C}$ joins $\left(\lambda_{1}, 0\right)$ to $(+\infty, 1)$. From Theorem 2.1 , we can see that $v$ is nonnegative for any $(\lambda, v) \in \mathscr{C}$. Define

$$
F(\lambda, v)=\lambda N r^{N-1}\left(\frac{f^{\prime}\left(\varphi^{-1}(v)\right)}{\sqrt{1-v^{\prime 2}}}-f\left(\varphi^{-1}(v)\right) H\left(\varphi^{-1}(v), r\right)\right)+\left(r^{N-1} \phi\left(v^{\prime}\right)\right)^{\prime}
$$

for any $(\lambda, v) \in \mathbb{R} \times \mathcal{B}_{1}(0)$ with $v \geq 0, \not \equiv 0$. Then, by some simple calculations, we have that

$$
F_{v}(\lambda, 0) v=\lim _{t \rightarrow 0^{+}} \frac{F(\lambda, t v)}{t}=\lambda r^{N-1} v+\left(r^{N-1} v^{\prime}\right)^{\prime} .
$$

So, if $(\mu, 0)$ is a bifurcation point of nonnegative solution set of problem (1.12), one has $\mu=\lambda_{1}$. It follows that $\mathscr{C} \cap\left(\left(\mathbb{R} \backslash\left\{\lambda_{1}\right\}\right) \times\{0\}\right)=\emptyset$. Furthermore, by Proposition 3.3 of [3], we have that $v$ is positive and strictly decreasing for any $(\lambda, v) \in \mathscr{C} \backslash\left\{\left(\lambda_{1}, 0\right)\right\}$.

(b) Clearly, one has that $(0,0) \in \mathscr{C}$. By Theorem 2.1 of [11] again, we know that $\mathscr{C}$ is connected. So, we have that $\mathscr{C}$ joins $(0,0)$ to $(+\infty, 1)$. In view of Proposition 3.3 of [3], it is sufficient to show that $\mathscr{C} \cap((0,+\infty) \times\{0\})=\emptyset$. Otherwise, there exists a sequence $\left(\lambda_{n}, v_{n}\right) \in \mathscr{C}$ such that $\left(\lambda_{n}, v_{n}\right) \rightarrow(\mu, 0)$ with $v_{n} \not \equiv 0$ for some $\mu>0$ as $n \rightarrow+\infty$. By Proposition 3.3 of [3] and Theorem 2.1, we have that $v_{n}>0$ and is decreasing in $[0, R)$.

Since $f_{0}+H_{0}=+\infty$, we have that

$$
\lambda_{n} N\left(1-v_{n}^{\prime 2}\right)^{3 / 2} \frac{f^{\prime}\left(\varphi^{-1}\left(v_{n}\right)\right)-f\left(\varphi^{-1}\left(v_{n}\right)\right) H\left(\varphi^{-1}\left(v_{n}\right), r\right)}{v_{n}}>\lambda_{1} \text { for any } r \in(0, R)
$$

and $n$ large enough. Since $v_{n}$ is decreasing, we have that

$$
\lambda_{n} N\left(1-v_{n}^{\prime 2}\right)^{3 / 2} \frac{f^{\prime}\left(\varphi^{-1}\left(v_{n}\right)\right)-f\left(\varphi^{-1}\left(v_{n}\right)\right) H\left(\varphi^{-1}\left(v_{n}\right), r\right)}{v_{n}}-\frac{N-1}{r} v_{n}^{\prime 3}>\lambda_{1}
$$

for any $r \in(0, R)$ and $n$ sufficiently large. From [10], we know that

$$
\begin{aligned}
-\left(r^{N-1} v_{n}^{\prime}\right)^{\prime}= & \lambda_{n} N r^{N-1}\left(1-v_{n}^{\prime 2}\right)^{3 / 2}\left(\frac{f^{\prime}\left(\varphi^{-1}\left(v_{n}\right)\right)}{\sqrt{1-v_{n}^{\prime 2}}}-f\left(\varphi^{-1}\left(v_{n}\right)\right) H\left(\varphi^{-1}\left(v_{n}\right), r\right)\right) \\
& -(N-1) r^{N-2} v_{n}^{\prime 3} .
\end{aligned}
$$

Applying the Sturm comparison theorem [6, Lemma 3], $v_{n}$ has at least a zero in $(0, R)$, which is a contradiction.

(c) It is easy to see that $(+\infty, 0) \in \mathscr{C}$ and $(+\infty, 1) \in \mathscr{C}$. Applying Lemma 3.1 of [12], we obtain that $\mathscr{C}$ is connected. Again by Proposition 3.3 of [3], it is enough to show that $\mathscr{C} \cap([0,+\infty) \times\{0\})=\emptyset$. Suppose, by contradiction, that there exists a sequence $\left(\lambda_{n}, v_{n}\right) \in \mathscr{C}$ with $v_{n} \not \equiv 0$ such that $\left(\lambda_{n}, v_{n}\right) \rightarrow(\mu, 0)$ for some $\mu \geq 0$ as $n \rightarrow+\infty$. As that of (b), one has that $v_{n}$ is positive and decreasing. 
Let $w_{n}=v_{n} /\left\|v_{n}\right\|$. We can see that $w_{n}$ satisfies

$$
\begin{aligned}
-w_{n}^{\prime \prime}= & \lambda_{n} N\left(1-v_{n}^{\prime 2}\right)^{3 / 2}\left(\frac{f^{\prime}\left(\varphi^{-1}\left(v_{n}\right)\right)}{v_{n} \sqrt{1-v_{n}^{\prime 2}}} w_{n}-\frac{f\left(\varphi^{-1}\left(v_{n}\right)\right) H\left(\varphi^{-1}\left(v_{n}\right), r\right)}{v_{n}} w_{n}\right) \\
& +\frac{(N-1)}{r}\left(1-v_{n}^{\prime 2}\right) w_{n}^{\prime}:=g_{n}(r) .
\end{aligned}
$$

Since $\left\|w_{n}\right\|=1, v_{n} \rightarrow 0$ in $X$ and $f_{0}+H_{0}=0$, we have that

$$
\left(1-v_{n}^{\prime 2}\right)^{3 / 2}\left(\frac{f^{\prime}\left(\varphi^{-1}\left(v_{n}\right)\right)}{v_{n} \sqrt{1-v_{n}^{\prime 2}}} w_{n}-\frac{f\left(\varphi^{-1}\left(v_{n}\right)\right) H\left(\varphi^{-1}\left(v_{n}\right), r\right)}{v_{n}} w_{n}\right) \rightarrow 0
$$

as $n \rightarrow+\infty$. For any $a \in(0, R]$ and any $r \in[a, R]$, we see that

$$
\left|\frac{(N-1)}{r}\left(1-v_{n}^{\prime 2}\right) w_{n}^{\prime}\right| \leq \frac{(N-1)}{a} .
$$

It follows that

$$
\left|w_{n}^{\prime \prime}\right| \leq \frac{(N-1)}{a}+1
$$

for any $r \in[a, R]$ and sufficiently large $n$. So, $w_{n}$ converges to some $w_{0}$ in $C^{1}[a, R]$. Hence, for each $r \in(0, R]$, we have that

$$
\lim _{n \rightarrow+\infty} g_{n}(r)=\frac{(N-1) w_{0}^{\prime}}{r}
$$

Integrating equation (3.5) from $r$ to $R$, we get that

$$
w_{n}^{\prime}(r)-w_{n}^{\prime}(R)=\int_{r}^{R} g_{n}(\tau) d \tau
$$

The Lebesgue Dominated Convergence Theorem implies that

$$
w_{0}^{\prime}(r)-w_{0}^{\prime}(R)=\int_{r}^{R} \frac{(N-1) w_{0}^{\prime}}{\tau} d \tau
$$

It follows that

$$
-\left(r^{N-1} w_{0}^{\prime}\right)^{\prime}=0, r \in(0, R) .
$$

We claim that $w_{0}^{\prime}(0)=0$. Integrating equation (3.5) from 0 and $R$, we obtain that

$$
\begin{aligned}
& \int_{0}^{R} \frac{(N-1)}{r}\left(1-v_{n}^{\prime 2}\right) w_{n}^{\prime} d r=-w_{n}^{\prime}(R)- \\
& \int_{0}^{R} \lambda_{n} N\left(1-v_{n}^{\prime 2}\right)^{3 / 2}\left(\frac{f^{\prime}\left(\varphi^{-1}\left(v_{n}\right)\right)}{v_{n} \sqrt{1-v_{n}^{\prime 2}}} w_{n}-\frac{f\left(\varphi^{-1}\left(v_{n}\right)\right) H\left(\varphi^{-1}\left(v_{n}\right), r\right)}{v_{n}} w_{n}\right) d r .
\end{aligned}
$$

It follows that

$$
\left|\int_{0}^{R} \frac{(N-1)}{r}\left(1-v_{n}^{\prime 2}\right) w_{n}^{\prime} d r d r\right| \leq C
$$


for some positive constant $C$. By the monotonicity of $w_{n}$, we derive that

$$
\int_{0}^{R} \frac{(N-1)}{r}\left(1-v_{n}^{\prime 2}\right)\left|w_{n}^{\prime}\right| d r=\left|\int_{0}^{R} \frac{(N-1)}{r}\left(1-v_{n}^{\prime 2}\right) w_{n}^{\prime} d r d r\right| \leq C .
$$

So,

$$
\left\{-(N-1)\left(1-v_{n}^{\prime 2}\right) \frac{w_{n}^{\prime}}{r}\right\}
$$

is a set of positive integrable functions. From the Fatou Lemma, we get that $-(N-1) w_{0}^{\prime} / r$ is also integrable on $[0, R]$. For any $r \in(0, R]$, integrating equation (3.6) from $r$ to $R$, we have that

$$
w_{0}^{\prime}(r)-w_{0}^{\prime}(R)=\int_{r}^{R}(N-1) \frac{w_{0}^{\prime}}{\tau} d \tau .
$$

Since $w_{0}^{\prime} / r$ is integrable on $[0, R]$, the limit of the right member exists when $r$ tends to 0 . It implies the existence of $\lim _{r \rightarrow 0^{+}} w_{0}^{\prime}(r)$. Noting the integrability of $w_{0}^{\prime} / r$, one has that $\lim _{r \rightarrow 0^{+}} w_{0}^{\prime}(r)=0$.

Clearly, we have $w_{0}(R)=0$. Therefore, we obtain

$$
\left\{\begin{array}{l}
-\left(r^{N-1} w_{0}^{\prime}\right)^{\prime}=0, r \in(0, R) \\
w_{0}^{\prime}(0)=w_{0}(R)=0
\end{array}\right.
$$

It follows that $w_{0} \equiv 0$, which contradicts the fact of $\left\|w_{0}\right\|=1$.

\section{Appendix: Deduction of equation (1.5)}

To the best of our knowledge, equation (1.5) was first given in [1] without derivation process. For the convenience of readers and the integrity of the paper, here we include the detailed derivation.

Let $e_{i}, i=1, \ldots, N$, denote the natural basis of $\mathbb{R}^{N}$. Choose $e_{N+1}$ such that

$$
\left\langle e_{N+1}, e_{i}\right\rangle= \begin{cases}-1 & \text { if } i=N+1 \\ 0 & \text { if } i \in\{1, \ldots, N\}\end{cases}
$$

where $\langle\cdot, \cdot\rangle$ denote the inner product of $\mathcal{M}$. Then

$$
f e_{1}, \ldots, f e_{N}, e_{N+1}
$$

are the natural basis of $\mathcal{M}$.

We assume that $u \in C^{2}(\Omega)$ and let $M=\{(u(x), x): x \in \Omega\}$. Then we have coordinates $\left(x_{1}, \ldots, x_{N}\right)$ on $M$ with coordinate tangent vectors $X_{i}=f e_{i}+u_{i} e_{N+1}$, where $u_{i}=\nabla_{i} u=$ $\partial u / \partial x_{i}, i=1, \ldots, N$. Then, the induced metric on $M$ is

$$
g_{i j}=\left\langle X_{i}, X_{j}\right\rangle=f^{2}(u) \delta_{i j}-u_{i} u_{j}, i, j \in\{1, \ldots, N\},
$$

where $\delta_{i j}=1(0)$ if $j=i(j \neq i)$. 
We claim that $\operatorname{det}\left(g_{i j}\right)=f^{2(N-1)}(u)\left(f^{2}(u)-|\nabla u|^{2}\right)$. Indeed, we have that

$$
\begin{aligned}
\operatorname{det}\left(g_{i j}\right) & =\left|f^{2}(u) E_{N}-\left(\begin{array}{c}
u_{1} \\
\vdots \\
u_{N}
\end{array}\right)\left(\begin{array}{lll}
u_{1} & \cdots & u_{N}
\end{array}\right)\right| \\
& =f^{2(N-1)}(u)\left|f^{2}(u)-\left(\begin{array}{lll}
u_{1} & \cdots & u_{N}
\end{array}\right)\left(\begin{array}{c}
u_{1} \\
\vdots \\
u_{N}
\end{array}\right)\right| \\
& =f^{2(N-1)}(u)\left(f^{2}(u)-|\nabla u|^{2}\right),
\end{aligned}
$$

where $E_{N}$ is the $N$-order identity matrix. In above, we have used the following elementary relations in advanced algebra

$$
\left|\begin{array}{cc}
E_{m} & B \\
A & E_{n}
\end{array}\right|=\left|E_{n}-A B\right|=\left|E_{m}-B A\right|
$$

and

$$
\left|\lambda E_{n}-A B\right|=\lambda^{n-m}\left|\lambda E_{m}-B A\right|,
$$

where $\lambda \neq 0, A$ and $B$ are $n \times m$ and $m \times n$ matrixes, respectively.

Take

$$
v=f(u) \sum_{i}^{N} v_{i} e_{i}+v_{N+1} e_{N+1}
$$

where

$$
v_{i}=\frac{u_{i}}{f(u) \sqrt{f^{2}(u)-|\nabla u|^{2}}}, i \in\{1, \ldots, N\}, v_{N+1}=\frac{f(u)}{\sqrt{f^{2}(u)-|\nabla u|^{2}}} .
$$

It is not difficult to verify that $v$ is the upward unit normal vector field to $M$. Some simple computations show that the adjoint matrix of $\left(g_{i j}\right)$ is

$$
\left(g_{i j}^{*}\right)=f^{2(N-2)}(u)\left(\left(f^{2}(u)-|\nabla u|^{2}\right) \delta_{j i}+u_{j} u_{i}\right) .
$$

It follows that the inverse of $\left(g_{i j}\right)$ is $\left(g^{i j}\right)$ with

$$
g^{i j}=\frac{g_{i j}^{*}}{\operatorname{det}\left(g_{i j}\right)}=\frac{\delta_{i j}}{f^{2}(u)}+v_{i} v_{j}, i, j \in\{1, \ldots, N\} .
$$


By Proposition 7.35 of [21], the second fundamental form can be calculated by

$$
\begin{aligned}
& A_{i j}=\left\langle X_{i}, \bar{\nabla}_{X_{j}} v\right\rangle=\left\langle X_{i}, \bar{\nabla}_{X_{j}}\left(\frac{1}{\sqrt{f^{2}(u)-|\nabla u|^{2}}}\left(\sum_{i}^{N} u_{i} e_{i}+f(u) e_{N+1}\right)\right)\right\rangle \\
& =\left\langle X_{i}, \bar{\nabla}_{X_{j}}\left(\frac{1}{\sqrt{f^{2}(u)-|\nabla u|^{2}}}\right)\left(\sum_{i}^{N} u_{i} e_{i}+f(u) e_{N+1}\right)\right\rangle+ \\
& \left\langle X_{i}, \frac{1}{\sqrt{f^{2}(u)-|\nabla u|^{2}}} \bar{\nabla}_{X_{j}}\left(\sum_{i}^{N} u_{i} e_{i}+f(u) e_{N+1}\right)\right\rangle \\
& =\left\langle X_{i}, \frac{1}{\sqrt{f^{2}(u)-|\nabla u|^{2}}} \bar{\nabla}_{X_{j}}\left(f(u) e_{N+1}\right)\right\rangle+\left\langle X_{i}, \frac{1}{\sqrt{f^{2}(u)-|\nabla u|^{2}}} \bar{\nabla}_{X_{j}}\left(\sum_{i}^{N} u_{i} e_{i}\right)\right\rangle \\
& =\left\langle X_{i}, \frac{f^{\prime}(u) X_{j}}{\sqrt{f^{2}(u)-|\nabla u|^{2}}}\right\rangle+ \\
& \frac{1}{\sqrt{f^{2}(u)-|\nabla u|^{2}}}\left\langle X_{i}, \frac{1}{f(u)} \bar{\nabla}_{X_{j}}\left(\sum_{i}^{N} u_{i} e_{i}\right)+\bar{\nabla}_{X_{j}}\left(\frac{1}{f(u)}\right)\left(\sum_{i}^{N} u_{i} e_{i}\right)\right\rangle \\
& =\frac{f^{2}(u) f^{\prime}(u) \delta_{i j}}{\sqrt{f^{2}(u)-|\nabla u|^{2}}}+\frac{f(u) u_{i j}}{\sqrt{f^{2}(u)-|\nabla u|^{2}}}-\frac{2 f^{\prime}(u) u_{i} u_{j}}{\sqrt{f^{2}(u)-|\nabla u|^{2}}},
\end{aligned}
$$

where $\bar{\nabla}$ is the Levi-Civita connection and $u_{i j}=\partial^{2} u / \partial x_{i} \partial x_{j}$.

Therefore, the mean curvature of $M$ is

$$
\begin{aligned}
& H=\frac{1}{N} \sum_{i, j=1}^{N} g^{i j} A_{i j} \\
& =\frac{1}{N} \sum_{i, j=1}^{N}\left(\frac{\delta_{i j}}{f^{2}(u)}+v_{i} v_{j}\right)\left(\frac{f^{2}(u) f^{\prime}(u) \delta_{i j}}{\sqrt{f^{2}(u)-|\nabla u|^{2}}}+\frac{f(u) u_{i j}}{\sqrt{f^{2}(u)-|\nabla u|^{2}}}-\frac{2 f^{\prime}(u) u_{i} u_{j}}{\sqrt{f^{2}(u)-|\nabla u|^{2}}}\right) \\
& =\frac{1}{N f(u) \sqrt{f^{2}(u)-|\nabla u|^{2}}} \sum_{i, j=1}^{N}\left(\delta_{i j}+\frac{u_{i} u_{j}}{f^{2}(u)-|\nabla u|^{2}}\right) u_{i j}+ \\
& \frac{f^{\prime}(u)}{\sqrt{f^{2}(u)-|\nabla u|^{2}}}-\frac{f^{\prime}(u)|\nabla u|^{2}}{N\left(f^{2}(u)-|\nabla u|^{2}\right)^{3 / 2}} \text {. }
\end{aligned}
$$

It follows that

$$
\operatorname{div}\left(\frac{\nabla u}{f(u) \sqrt{f^{2}(u)-|\nabla u|^{2}}}\right)+\frac{f^{\prime}(u)}{\sqrt{f^{2}(u)-|\nabla u|^{2}}}\left(N+\frac{|\nabla u|^{2}}{f^{2}(u)}\right)=N H,
$$

which is just equation (1.5).

\section{References}

[1] L.J. Alías, A. Romero and M. Sánchez, Uniqueness of complete spacelike hypersurfaces of constant mean curvature in Generalized Robertson-Walker spacetimes, Gen. Relativity Gravitation 27 (1995), 71-84. 
[2] R. Bartnik and L. Simon, Spacelike hypersurfaces with prescribed boundary values and mean curvature, Comm. Math. Phys. 87 (1982), 131-152.

[3] C. Bereanu, D. de la Fuente, A. Romero and P.J. Torres, Existence and multiplicity of entire radial space like graphs with prescribed mean curvature function in certain Friedmann-Lemaître-Robertson-Walker space times, Commun. Contemp. Math. 19 (2017), 1-18.

[4] C. Bereanu, P. Jebelean and P.J. Torres, Positive radial solutions for Dirichlet problems with mean curvature operators in Minkowski space, J. Funct. Anal. 264 (2013), 270287.

[5] C. Bereanu, P. Jebelean and P.J. Torres, Multiple positive radial solutions for a Dirichlet problem involving the mean curvature operator in Minkowski space, J. Funct. Anal. 265 (2013), 644-659.

[6] H. Berestycki, On some nonlinear Sturm-Liouville problems, J. Differential Equations 26 (1977), 375-390.

[7] E. Calabi, Examples of Berstein problems for some nonlinear equations, Proc. Sympos. Pure Math. Amer. Math. Soc, 15 (1970), 223-230.

[8] S.-Y. Cheng and S.-T. Yau, Maximal spacelike hypersurfaces in the Lorentz-Minkowski spaces, Ann. of Math. 104 (1976), 407-419.

[9] Y. Choquet-Bruhat, General Relativity and the Einstein Equations, Oxford University Press, 2009.

[10] G. Dai, Bifurcation and positive solutions for problem with mean curvature operator in Minkowski space, Calc. Var. Partial Differential Equations 55 (2016), paper 72, 17 pp.

[11] G. Dai, Two Whyburn type topological theorems and its applications to Monge-Ampére equations, Calc. Var. Partial Differential Equations 55 (2016), paper 97, 28 pp.

[12] G. Dai, Bifurcation and nonnegative solutions for problem with mean curvature operator on general domain, Indiana Univ. Math. J., http://www.iumj.indiana.edu /IUMJ/Preprints/7546.pdf.

[13] K. Deimling, Nonlinear Functional Analysis, Springer-Verlag, New-York, 1987.

[14] A. Friedmann, On the curvature of space, Translated from the 1922 German original, Gen. Relativity Gravitation 31 (1999), 1991-2000.

[15] A. Friedmann, On the possibility of a world with constant negative curvature of space, Translated from the 1924 German original, Gen. Relativity Gravitation 31 (1999), 20012008.

[16] D. de la Fuente, A. Romero and P.J. Torres, Radial solutions of the Dirichlet problem for the prescribed mean curvature equation, Adv. Nonlinear Stud. 15 (2014), 171-181.

[17] E.L. Ince, Ordinary Differential Equation, Dover Publication Inc. New York, 1927. 
[18] G. Lemaître, Republication of: A homogeneous universe of constant mass and increasing radius accounting for the radial velocity of extra-galactic nebulae, Originally published in Ann. Soc. Sci. Bruxelles 47A (1927), 49-59, and translated from the French by Jean-Pierre Luminet, Gen. Relativity Gravitation 45 (2013), 1635-1646.

[19] R. Manásevich and J. Mawhin, Periodic Solutions for Nonlinear Systems with pLaplacian-Like Operators, J. Differential Equations 145 (1998), 367-393.

[20] J. Mawhin and P.J. Torres, Prescribed mean curvature graphs with Neumann boundary conditions in some FLRW spacetimes, J. Differential Equations 261 (2016), 7145-7156.

[21] B. O'Neill, Semi-Riemannian Geometry with application to Relativity, Academic Press, 1983.

[22] R. Osserman, The minimal surface equation, Seminar on nonlinear partial differential equations, Math. Sci. Res. Inst. Publ., Springer, New York, Vol 2 (1984), 237-259.

[23] P.H. Rabinowitz, Some global results for nonlinear eigenvalue problems, J. Funct. Anal. 7 (1971), 487-513.

[24] P.H. Rabinowitz, On bifurcation from infinity, J. Funct. Anal. 14 (1973), 462-475.

[25] H.P. Robertson, Kinematics and world structure, Astrophys. J. 82 (1935), 284-301.

[26] H.P. Robertson, Kinematics and world structure II, Astrophys. J. 83 (1936), 187-201.

[27] H.P. Robertson, Kinematics and world structure III, Astrophys. J. 83 (1936), 257-271.

[28] K. Schmitt, R. Thompson, Nonlinear Analysis and Differential Equations: An Introduction, Univ. of Utah Lecture Notes, Univ. of Utah Press, Salt Lake City, 2004.

[29] A.E. Treibergs, Entire spacelike hypersurfaces of constant mean curvature in Minkowski space, Invent. Math. 66 (1982), 39-56.

[30] A.G. Walker, On Milne's theory of world-structure, Proc. Lond. Math. Soc. (2) 42 (1937), 90-127.

[31] W. Walter, Ordinary Differential Equations, Springer, New York, 1998.

[32] G.T. Whyburn, Topological Analysis, Princeton University Press, Princeton, 1958. 